\title{
SIR-2.1 integrates metabolic homeostasis with the reproductive neuromuscular excitability in early aging male Caenorhabditis elegans
}

\author{
Xiaoyan Guo1, L René García ${ }^{1,2 *}$ \\ 'Department of Biology, Texas A\&M University, College Station, United States; \\ ${ }^{2}$ Howard Hughes Medical Institute, Texas A\&M University, Texas, United States
}

\begin{abstract}
The decline of aging $C$. elegans male's mating behavior is correlated with the increased excitability of the cholinergic circuitry that executes copulation. In this study, we show that the mating circuits' functional durability depends on the metabolic regulator SIR-2.1, a NAD+-dependent histone deacetylase. Aging sir-2.1(0) males display accelerated mating behavior decline due to premature hyperexcitability of cholinergic circuits used for intromission and ejaculation. In sir-2.1(0) males, the hypercontraction of the spicule-associated muscles pinch the vas deferens opening, thus blocking sperm release. The hyperexcitability is aggravated by reactive oxygen species (ROS). Our genetic, pharmacological, and behavioral analyses suggest that in sir-2.1(0) and older wild-type males, enhanced catabolic enzymes expression, coupled with the reduced expression of ROSscavengers contribute to the behavioral decline. However, as a compensatory response to reduce altered catabolism/ROS production, anabolic enzymes expression levels are also increased, resulting in higher gluconeogenesis and lipid synthesis.
\end{abstract}

DOI: 10.7554/eLife.01730.001

*For correspondence: rgarcia@ bio.tamu.edu

Competing interests: The authors declare that no competing interests exist.

Funding: See page 19

Received: 18 October 2013 Accepted: 16 March 2014 Published: 22 April 2014

Reviewing editor: Andrew Dillin, Howard Hughes Medical Institute, UC Berkeley, United States

(c) Copyright Guo and García. This article is distributed under the terms of the Creative Commons Attribution License, which permits unrestricted use and redistribution provided that the original author and source are credited.

\section{Introduction}

Although lifespan is well studied in the model organism Caenorhabditis elegans (Kenyon, 2010), the aging process per se is still under intense research (Jin, 2010). For example, behavioral and/or mental ability starts to decline during early aging, prior to any dramatic structural or morphological dysfunction (Salthouse, 2004; Guo et al., 2012). The decline could be due to physiological alterations toward a non-optimal state, which leads to the failure of proper behavioral execution. Therefore, it is urgent to uncover the molecular mechanism(s) underlying the physiological changes, so that certain actions could prevent or postpone the non-optimal modifications that occur during aging.

Our previous studies used $C$. elegans males to establish a behavioral model for studying physiological alterations that occur during early aging (Guo et al., 2012). The spicule intromission motor step, which occurs during $C$. elegans male copulation, is sensitive to changes that occur during early aging (Garcia et al., 2001; Garcia and Sternberg, 2003). Through pharmacological, optogenetics, and genetic analyses, we found a correlation between the increased excitability of the spicule intromission circuit and the decline of mating at early adulthood (Guo et al., 2012). However, the molecular mechanisms underlying this correlation were unknown.

Caloric restriction is an effective way to extend lifespan (Hursting et al., 2003). In C. elegans, caloric deprivation such as transient starvation for 3 to $18 \mathrm{hr}$ during early adulthood can reduce excitability in the spicule intromission circuitry and prolong the mating potency of wild type (LeBoeuf et al., 2011). We showed that the effect of transient starvation is partially mediated by UNC-43/ CaMKII and up-regulation of the unc-103 and egl-2-encoded ERG-like $\mathrm{K}^{+}$channels (LeBoeuf et al., 2011; Guo et al., 2012). Although males with mutations in both $\mathrm{K}^{+}$channels abrogate most of the 
eLife digest Although the signs of aging are clear to us all, precisely why we age is less well understood. One possibility is that as cells use oxygen to fuel the breakdown of large molecules into smaller ones to release energy, they also generate by-products called reactive oxygen species that can damage DNA. As we get older, this damage gets worse. Consistent with this idea, it has been shown that a reduced calorie intake can reduce oxidative damage in certain species, in addition to extending lifespan.

Many experiments on aging have been performed on worms belonging to the species C. elegans. Male worms of this species live for an average of 11-12 days, but begin to show signs of aging - for example, a reduced ability to mate-as early as day 3 of their adult lives. Now, Guo and García have revealed that a protein called SIR-2.1, which regulates metabolism in worms, also helps to protect the animals from the effects of aging.

Male worms in which the gene for this protein has been 'knocked out' have a normal lifespan, but show signs of aging earlier than normal males. They are also more susceptible to the damaging effects of reactive oxygen species, suggesting that SIR-2.1 may offer protection against oxidative damage. Indeed, levels of ATP — the molecule used to move energy around inside cells_are increased in knockout worms. This suggests that certain metabolic processes and the production of reactive oxygen species, are increased in the knockout worms, which speeds up the aging process.

While the link between metabolism and aging is well known, the work of Guo and García offers insights into some of the molecular mechanisms that may form the basis of this relationship.

DOI: $10.7554 /$ Life. 01730.002

beneficial effects of transient starvation, we still observed a small increase in the mating ability of aged double-mutant males, suggesting that other mechanisms are applied by transient starvation to improve mating (Guo et al., 2012). One possibility is that metabolic alteration, induced by transient starvation, compensates for the physiological changes that occur during aging. The connection between metabolic change and physiological status might be the generation of reactive oxygen species (ROS). First, metabolic status determines the oxidative stress burden: ROS is the byproduct of oxidative phosphorylation (Murphy, 2009). Second, emerging evidence shows that ROS modulates the excitability of neuromuscular system through modification of ion channels (Taglialatela et al., 1997; Cai and Sesti, 2009; Aggarwal and Makielski, 2013). However, there are few comprehensive in vivo studies, which link ROS-mediated physiological changes to complex behavior alteration.

To address this, we investigated how a mutation in the C. elegans metabolism regulator, SIR-2.1, alters behavior. SIR-2.1 is an ortholog of yeast SIR2 (Tissenbaum and Guarente, 2001). Yeast SIR2, a $\mathrm{NAD}^{+}$-dependent histone deacetylase, with a role in chromatin regulation, can silence ribosome DNA expression and recombination (Gottlieb and Esposito, 1989). When overexpressed, sir2 extends yeast lifespan, whereas deletion of the gene shortens the lifespan by $50 \%$ (Kaeberlein et al., 1999). Although there is controversy on whether overexpression of invertebrate sir2 ortholog extends lifespan (Burnett et al., 2011; Viswanathan and Guarente, 2011), sirtuin family proteins have been shown to regulate glucose and fat metabolism (Houtkooper et al., 2012). For example, the C. elegans SIR-2.1 inhibits lipid synthesis during fasting (Walker et al., 2010). In addition, sirtuin proteins mediate an oxidative stress response by regulating antioxidant gene expression through transcriptional factors such as FOXO in a 14-3-3-dependent manner (Berdichevsky et al., 2006; Webster et al., 2012; Merksamer et al., 2013). Overall, sirtuin proteins could be involved in age-related diseases, such as type II diabetes and neurodegenerative diseases (Satoh et al., 2011; Houtkooper et al., 2012). Taken together, it is possible that sirtuin proteins regulate the cell's metabolic status and physiology, which further alters the coordination of specific behaviors.

In this study, we used $C$. elegans male mating behavior to study the molecular and physiological alterations underlying the behavioral decline that occur during early aging. We found that wild-type males require SIR-2.1 to maintain mating potency, and sir-2.1 mutant males show a premature decline in copulation behavior, consistent with oxidative stress-induced hyperexcitability of their mating circuit. We propose that in sir-2.1(0) males, enhanced glycolysis/fatty acid oxidation, coupled with a compromised anti-stress system, contribute to premature mating decline. However, in mutant and aged wild-type males, pyruvate carboxylase and phosphenolpyruvate carboxykinase are up-regulated, 
likely as a compensatory mechanism to shunt excess pyruvate from glycolysis and the TCA cycle to lipid biosynthesis, gluconeogenesis, and glyceroneogenesis.

\section{Results}

\section{SIR-2.1 maintains male mating during early aging}

Previously, we reported that $C$. elegans male mating behavior deteriorates during early aging. N2 and him-5(e1490) C. elegans (henceforth, will be referred to as wild type) males' mating capability begins to decline at day 3 of their adulthood, although their median lifespan is 11-12 days (Guo et al., 2012). We demonstrated that transient starvation of young males can extend their mating span, partially through up-regulation of ether-a-go-go $\mathrm{K}^{+}$channels (LeBoeuf et al., 2011); however, our data also suggested that transient starvation can improve mating through additional mechanisms (Guo et al., 2012). Considering that metabolism is altered in food-deprived males (Tan et al., 2011), we tested whether perturbing the histone deacetylase metabolic regulator, sir-2.1, affects the functional span of copulation behavior in fed and transiently starved/re-fed males.

In adult hermaphrodites, animals that lack sir-2.1 have increased intestinal lipids (Walker et al., 2010), a phenotype opposite of starved animals. Likewise, we found that 1-day-old sir-2.1(ok434) null (0) males also contain more lipids than wild type (Figure 1A). In addition, we observed that 2-day-old wild-type males have more fat (Figure 1A). Thus, we asked if sir-2.1(0) males might have altered mating due to metabolic dysregulation. Allowing the males to mate for $5 \mathrm{hr}$, we found that well-fed aging sir-2.1(0) males' mating ability drops prematurely, compared to wild-type males (Figure 1B (i)). Even under unlimited mating conditions, the mating potency of 2-day-old sir-2.1(0) drops to $42 \%$ $(\mathrm{p}<0.0001, \mathrm{n}=47$ ) (Figure 1B (ii)).

We then asked if transient starvation can suppress the mating defect in sir-2.1(0). To do so, we starved sir-2.1(0) males for $\sim 20 \mathrm{hr}$ from L4, and conducted a $5 \mathrm{hr}$ mating potency assay. Transient starvation improved mating of 2-day-old sir-2.1(0) males from $13 \%$ to $75 \%(p<0.0001$, Figure $1 \mathrm{C})$, but at day 3, the mating potency of transiently starved sir-2.1(0) males was still lower than wild type. Thus, similar to wild type, the metabolic alteration and/or up-regulated EAG $\mathrm{K}+$ channel functions caused by starvation alleviate some of the dysfunction caused by the sir-2.1 deletion. However, the mutant's reduced mating potencies between day 1 and 3 under both conditions indicate that SIR-2.1 contributes to the functionality of the mating circuits during this period.

To confirm that premature mating deterioration in sir-2.1(0) males is caused by the ok434 allele, we introduced into sir-2.1(0) animals a rescuing transgene containing the sir-2.1 endogenous promoter driving the sir-2.1 genomic sequence fused to $y$ fp. The extrachromosomal expression of sir-2.1 significantly improved the mating potency of 2-day-old sir-2.1(0) males from $26 \%$ to $75 \%(p<0.0001$, Figure 1D). sir-2.1 is expressed broadly in C. elegans (Bamps et al., 2009), thus we further conducted tissue-specific rescue assays, but found that none of the tissue-specific promoters driving the expression of sir-2.1, including neuronal, muscle, and intestinal promoters can rescue the premature mating decline (Figure1-figure supplement 1A), suggesting that sir-2.1 is required in multiple tissues to maintain male mating.

To exclude the possibility that sir-2.1(0) mating deficiency at day 2 is due to a shorter lifespan, we conducted a lifespan assay and found that sir-2.1(0) males lived as long as wild type (Figure 1-figure supplement 1B). Another possibility for the mating deterioration is morphological deformities of the sex musculature. However, in 2-day-old sir-2.1(0) males expressing a functional yfp:act-1 transgene (Figure 1-figure supplement 1C), we did not observe any obvious muscle fiber disorganization, which normally occurs in 8-day-old wild-type males (Guo et al., 2012). Although we did not inspect neural morphology, published studies showed that neural morphology does not change in $C$. elegans during aging (Herndon et al., 2002).

Another potential explanation for the lower mating efficiency is that sperm activity in 2-day-old sir-2.1(0) males is defective. C. elegans male sperm are stored in the seminal vesicle as a non-activated form and become activated after transfer into a hermaphrodite. Regulated by proteases, individual sperm goes through a morphological change to form a pseudopod. This pseudopod provides mobility for the sperm to fertilize the hermaphrodite oocyte (Smith and Stanfield, 2011). To test whether the low mating potency of 2-day-old sir-2.1(0) males is due to failure in sperm activation, we did an in vitro sperm activation assay and found that similar to wild type, $92.0 \pm 4.3 \%$ of sperms from 2-day-old sir-2.1(0) can be artificially activated by pronase (Figure 1-figure supplement 1D).Taken together, 
A

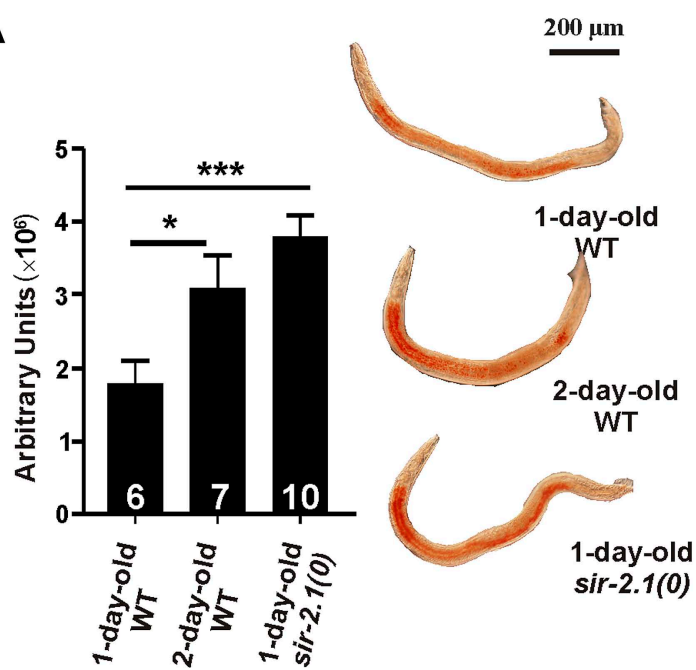

C

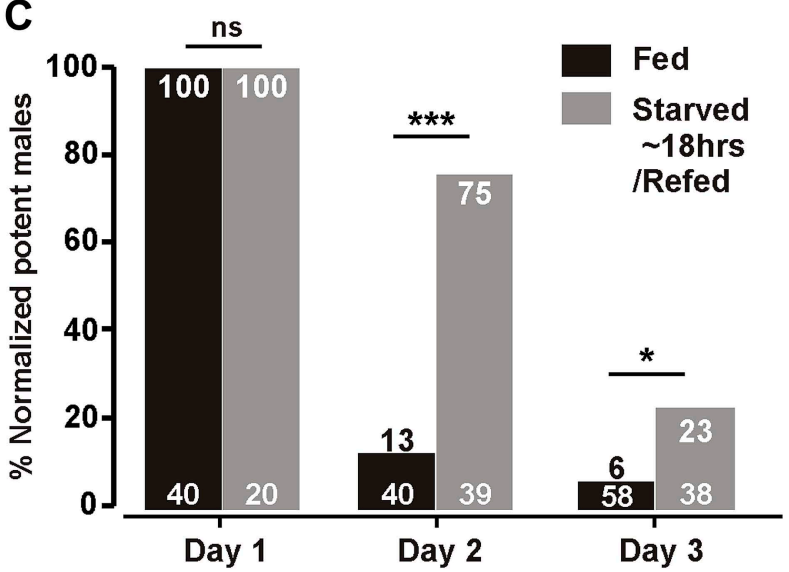

B

(i)

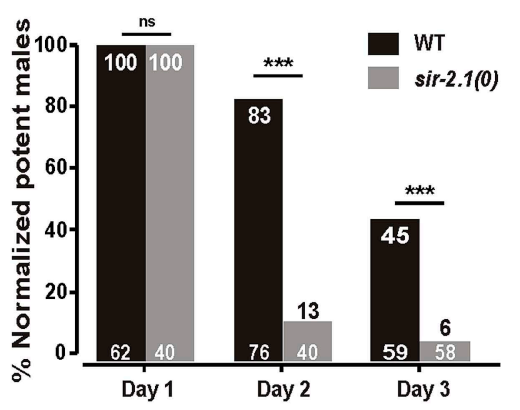

5 hrs mating

D

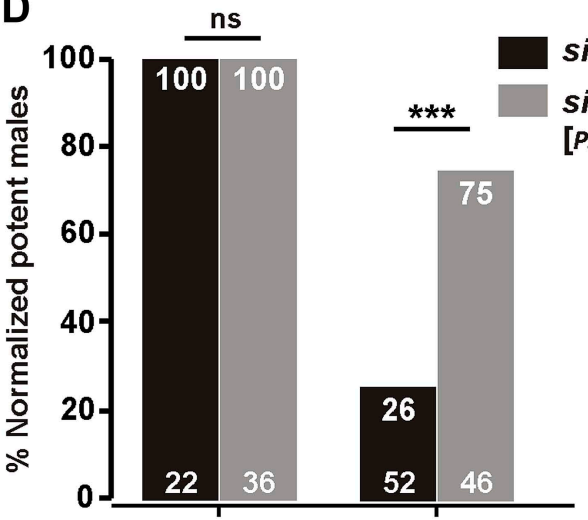

Day 1 (ii)

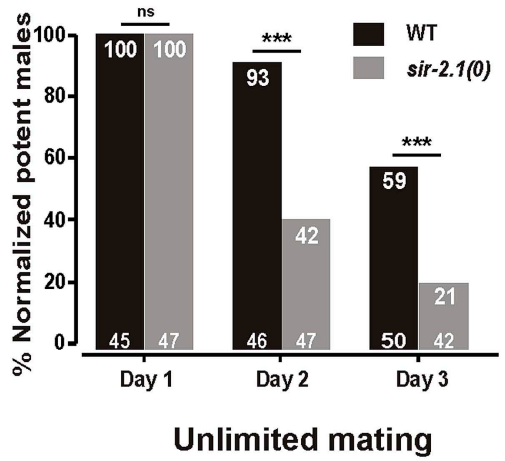

sir-2.1(0)

sir-2.1(0)

[Psir-2.1:sir-2.1::yfp]

Day 2

Figure 1. sir-2.1(0) males have altered lipid content and their mating ability deteriorates prematurely. (A) 1-day-old sir-2.1(0) and 2-day-old wild-type males have more lipid content than 1-day-old wild type. Left: quantification of fat staining, mean \pm SEM, unpaired t-test. Right: representative images of fat staining. (B) Mating potency of wild-type and sir-2.1(0) males. Copulations were allowed to occur for 5 hr (i) or for an unlimited time (ii). The number of males in each assay is listed at the bottom of each bar. The numerical percentage of wild-type males that mated on day 1 was normalized to $100 \%$. The normalization factor was then applied to the other experimental conditions. The normalized percentages for each day are listed on the top. Fisher's exact test was used to compare the mating potency prior to normalization. (C) Transient starvation reduces sir-2.1(0) mating deficiency. (D) Mating potency of sir-2.1(0) and rescued strain sir-2.1(0); rgEX399 [Psir-2.1:sir-2.1::yfp]. ns, not significant. Asterisks *, ** and ${ }^{\star \star \star}$ indicate the p<0.05, 0.01, and 0.0001 in this paper, respectively.

DOI: 10.7554/eLife.01730.003

The following figure supplements are available for figure 1:

Figure supplement 1. (A) Tissue specific expression of sir-2.1 does not rescue the reduced mating potency of sir-2.1(0) males at day 2 .

DOI: 10.7554/eLife.01730.004

we speculate that the premature mating decline in sir-2.1(0) is due to physiological changes, rather than the structural degeneration of either neuromuscular circuits or sperm.

\section{sir-2.1(0) males mating circuit becomes more excitable}

We showed that wild-type mating deterioration at day 3 is correlated with an increased excitability in the mating circuitry (Guo et al., 2012). Hence, we hypothesized that sir-2.1(0) mating decline might also be due to a premature increase in the cellular excitability. To test this, we used two acetylcholine (ACh) agonists, levemisole (LEV) and arecoline (ARE) to determine the responses of wild-type and sir-2.1(0) males at multiple ages. In the male spicule intromission circuit, LEV binds to ionotropic 
ACh receptors, whereas ARE is a nonselective ACh agonist (Liu et al., 2007; Correa et al., 2012). Activation of ACh receptors depolarizes the male's neurons and muscles, and ultimately causes sex muscle contractions; as a result, males protrude their copulatory spicules. We found that at day 1 , sir-2.1(0) and wild-type males had similar response to a sub-threshold effective concentration of ARE $(50 \mu \mathrm{M})$ (Figure 2A (i)). However, 2-day-old sir-2.1(0) males were more sensitive to agonist stimulation and required significantly less time to respond (Figure 2A (ii)). Additionally, 2-day-old sir-2.1(0) males were more sensitive to sub-threshold LEV stimulation. $58 \%$ sir- $2.1(0)$ compared to $35 \%$ of wild type protracted their spicules in $500 \mathrm{nM} \mathrm{LEV}(\mathrm{p}<0.05, \mathrm{n}>30)$ (Figure 2B (ii)). These results indicate that the loss of sir-2.1 in males alters the spicule intromission circuit's excitability during early aging.

\section{Hyperexcitability leads to an ejaculation defect}

To address how hyperexcitability disrupts copulation, we observed the mating behavior of 2-day-old sir-2.1(0) and wild-type males. We found that 2-day-old sir-2.1(0) males performed most of the mating steps similarly to the wild-type control (Figure 2- figures supplement $1 A, B, C, D$ ). Although 2-day-old sir-2.1(0) males can effectively insert their spicules, a significant number of them failed to transfer sperm into the hermaphrodite (Figure 2C). Upon spicule insertion, sperm moved out from the seminal vesicle and traveled through the vas deferens; however, they remained stuck in the vas deferens and did not drain through the cloacal opening (Videos 1, 2 and 3). Even the exceptional sir-2.1(0) males that successfully ejaculated, transferred less sperm and produced fewer progeny (Figure 2D).

The male copulatory spicules are attached to three sets of sex muscles: the retractor, protractor, and anal depressor muscles. Contraction of the protractor muscles leads to spicules insertion into the vulva (Garcia et al., 2001). During the normal ejaculation step of mating behavior, after spicule penetration, the posterior gubernaculum erector and retractor muscles contract, presumably to pull the proctodeum posteriorly, so that sperm can drain from the vas deferens (Figure 3-figure supplement 1) (Liu et al., 2007). In sir-2.1(0) males, we speculated that after spicule insertion, the abnormal increased cell excitability causes the spicule protractor and anal depressor muscles to hypercontract, which during sperm transfer, would pinch close the vas deferens opening. To test this, we imaged the $\mathrm{Ca}^{2+}$ in the spicule-associated dorsal protractor and anal depressor muscles (region-of-interest, $\mathrm{ROI}$, indicated in Figure 3A,B), by expressing G-CaMP3 in these sex muscles of both sir-2.1(0) and wildtype males (Tian et al., 2009; Guo et al., 2012). During the mating behavior of 2-day-old wild-type males, the G-CaMP3 $\triangle F / F 0$ increased to $129.0 \pm 32.5 \%(n=5)$ at the time of spicule insertion, and the $\mathrm{Ca}^{2+}$ signal started to decline to $86.7 \pm 30.8 \%$ during the 10 -s period after spicule insertion (Figure $3 \mathrm{~A}$ and Video 4). This indicates that the spicule protractor muscles partially relax after spicule insertion. However, in 2-day-old sir-2.1(0) males, $\Delta F / F 0$ increased up to $204.3 \pm 97.5 \%(n=5)$ upon spicule insertion. Unlike wild type, $\mathrm{Ca}^{2+}$ transients did not decrease as much, and the $\Delta \mathrm{F} / \mathrm{FO}$ fluctuated at about $129.8 \pm 33.0 \%$ (Figure 3B and Video 5). The sustained higher $\mathrm{Ca}^{2+}$ levels in 2-day-old sir-2.1(0) males suggest that spicule protractor and anal depressor muscles are hypercontracted and pinch the vas deferens opening, thus blocking sperm release.

\section{Reactive oxygen species leads to the mating deterioration}

C. elegans hermaphrodite studies showed that SIR-2.1 promotes the expression of antioxidant genes through its association with the FOXO/DAF-16 transcription factor (Berdichevsky et al., 2006). sir-2.1(0) hermaphrodites are more sensitive to stresses such as reactive oxygen species (ROS) (Rizki et alı, 2011). Therefore, we asked if ROS-induced damage contributes to the premature mating deterioration. We confirmed that similar to hermaphrodites, sir-2.1(0) males are also more sensitive to paraquat, a ROS-generator. Mutant males are less viable in $10 \mathrm{mM}$ paraquat after $24 \mathrm{hr}$; $89 \%$ of sir-2.1(0) males survived, compared to 99\% of wild type $(\mathrm{p}<0.01, \mathrm{n}>100)$ (Figure $4 \mathrm{~A})$. When exposure time reached $48 \mathrm{hr}$, the difference between two strains became more obvious, $39 \%$ of wild-type males survived, compared to $4 \%$ of sir-2.1(0) $(p<0.001)$ (Figure $4 A$ ).

Since sir-2.1(0) males are more sensitive to oxidative stress, we hypothesized that during aging, accumulated ROS from metabolism might contribute to the decreased mating efficiency and to the increased excitability of the spicule intromission circuit. To test this, we grew wild-type males on plates containing $1 \mathrm{mM}$ paraquat from late $\mathrm{L} 4$ to adult and assayed their mating ability and genital muscle excitability. After exposure to paraquat for $24 \mathrm{hr}$, males showed significant decline in mating potency (Figure 4B). Additionally, these males also displayed increased genital muscle sensitivity to the day 1 


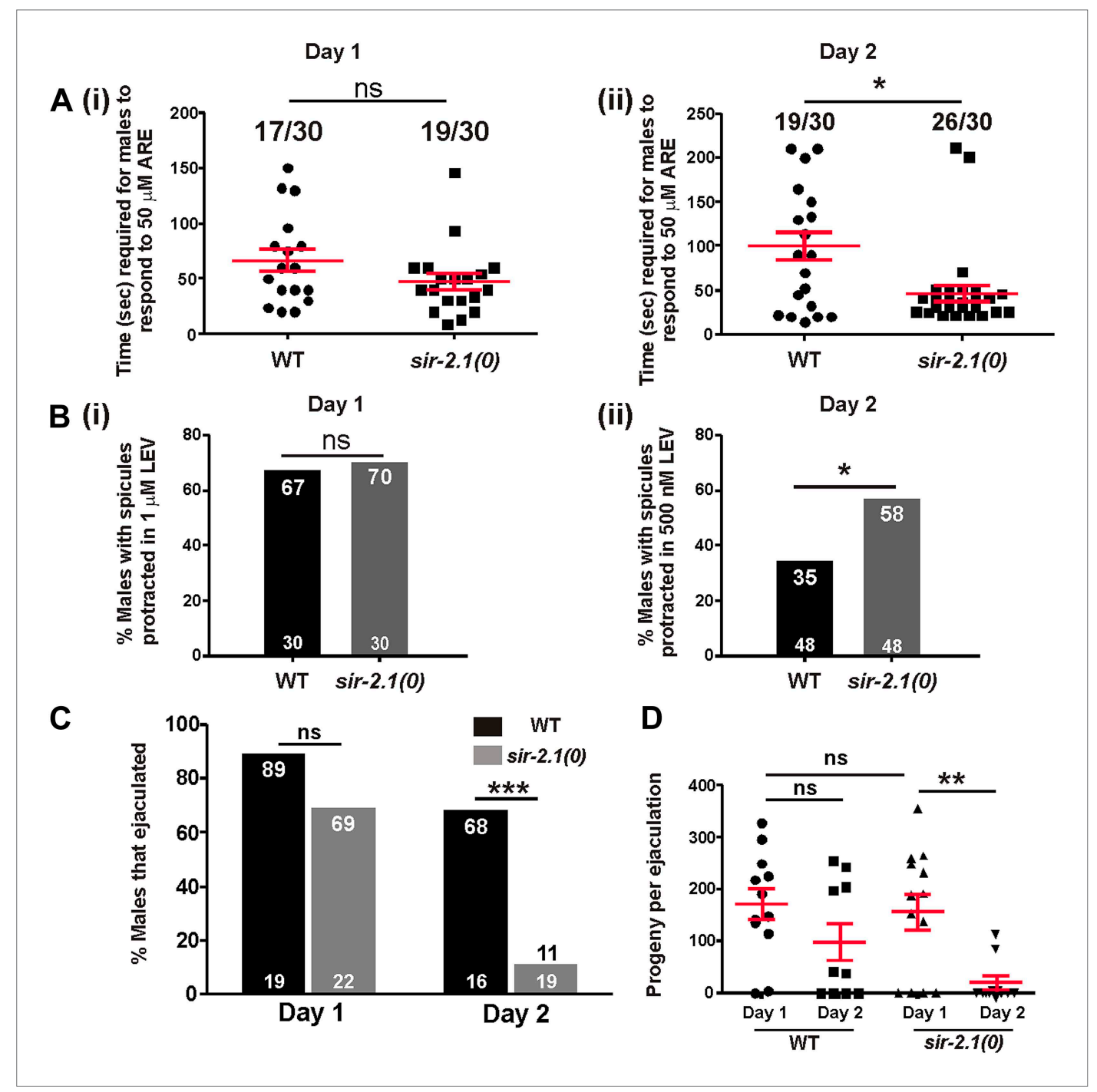

Figure 2. sir-2.1(0) males' sex circuitry becomes more excitable during aging, and those males display ejaculation defects. (A) 1-day-old wild-type and sir-2.1(0) males $(n=30)$ have similar response to the ACh agonist arecoline (ARE). The time required for those males to protrude their spicules out in $50 \mu \mathrm{M}$ ARE solution are not significantly different (i) (unpaired t-test), whereas 2-day-old sir-2.1(0) males require significantly less time to respond to ARE (ii) (unpaired t-test). Mean and SEM are indicated. (B) 1-day-old wild-type and sir-2.1(0) males $(n=30)$ have similar response to the ACh agonist levamisole (LEV) (i). However, 2-day-old sir-2.1(0) males are more sensitive to LEV (ii). (Fisher's exact test). (C, D) 2-day-old sir-2.1(0) males have an ejaculation defect. (C) The percentages of 2-day-old wild-type and sir-2.1(0) males that ejaculated during copulation. (Fisher's exact test). (D) The numbers of cross progeny produced by individual 2-day-old wild-type and sir-2.1(0) with unc-64(e240) hermaphrodites. Mean and SEM are indicated (unpaired t-test).

DOI: 10.7554/eLife. 01730.005

The following figure supplements are available for figure 2:

Figure supplement 1. (A) A cartoon illustration of $C$. elegans male mating behavior. DOI: 10.7554/eLife.01730.006

$\mathrm{EC}_{50}$ concentration $(1 \mu \mathrm{M})$ of LEV. $56 \%$ of wild type protracted their spicules; however, $83 \%$ males exposed to paraquat responded to the $A C h$ agonist $(p<0.05, n=54)$ (Figure $4 C$ ).

To further test if ROS contributes to the copulation decline, we supplemented the males' media with the antioxidant $\mathrm{N}$-acetyl-cysteine (NAC) (Schulz et al., 2007), and asked if NAC can delay genital muscle excitability changes and improve fertility. Indeed, when we exposed wild-type and sir-2.1(0) males to NAC from L4 to adulthood day 3 and adulthood day 2, respectively, the antioxidant decreased 


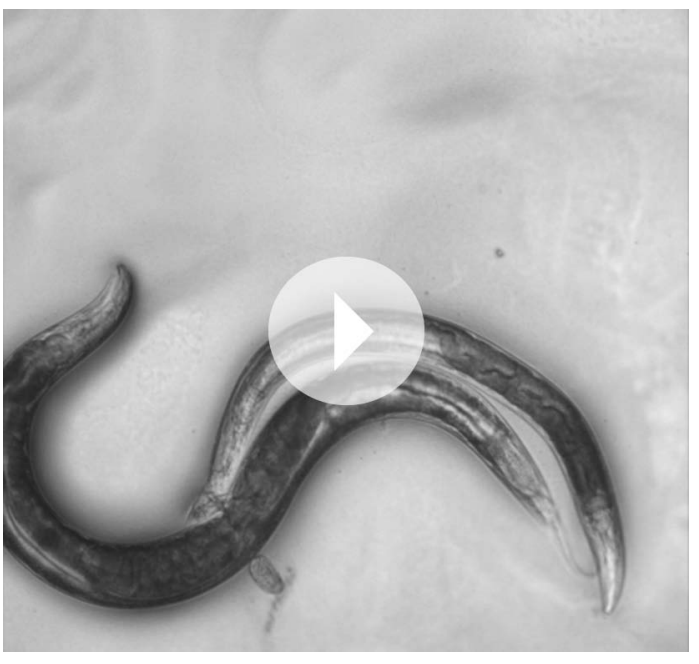

Video 1. Wild-type male's ejaculation. DOI: 10.7554/eLife.01730.007

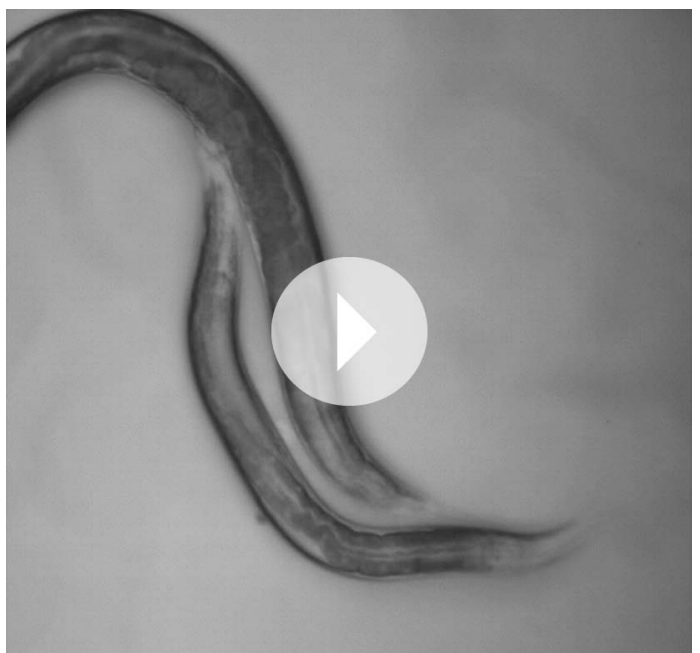

Video 2. 2-day-old sir-2.1(0) male's ejaculation DOI: 10.7554/eLife.01730.008

did not change in their levels (Supplementary file 1). In contrast, expression of ETC/OXPHOS components (cco-1, W09C5.8) was reduced in sir-2.1(0) (Figure 5F). Other genes that were significantly up-regulated include key anabolic enzymes like fatty acid desaturase (fat-5,6,7), pyruvate carboxylase (PC) (pyc-1) and phosphoenolpyruvate carboxykinase (PEPCK) (pck-1 and pck-2), isocytrate lysase (icl-1) and aconitase-cytosol (aco-1), which are important for fatty acid biosynthesis, gluconeogenesis, glyceroneogensis, and glyoxylate cycle (Figure 5D,E) (Yang et al., 2009). Consistent with this, hepatic cells without sirt1 also have an up-regualtion of PEPCK gene expression (Wang et alo, 2011). Fatty acid desaturase plays a critical role in lipid/triglyceride biosynthesis (Van Gilst et al., 2005; Flowers and Ntambi, 2008). PC catalyzes the carboxylation of pyruvate to oxaloacetate (OAA), the first step that shunts pyruvate from glycolysis to gluconeogenesis/glyceroneogenesis. Alternatively, OAA, an intermediate of TCA, can be converted to phosphoenolpyruvate (PEP) by PEPCK directly inside the mitochondrion or transported and converted to PEP by PEPCK in cytosol (Figure 6A). The up-regulation of fatty acid desaturase is consistent with the increased lipid staining in sir-2.1(0) males (Figure 1A).

To confirm if the changes in mRNA levels of those metabolic enzymes reflect functional alterations in the metabolic processes, we also measured ATP, glucose and glycogen accumulation in wild-type and sir-2.1(0) males. Consistent with increased expression levels of glycolysis and fatty acid oxidation 


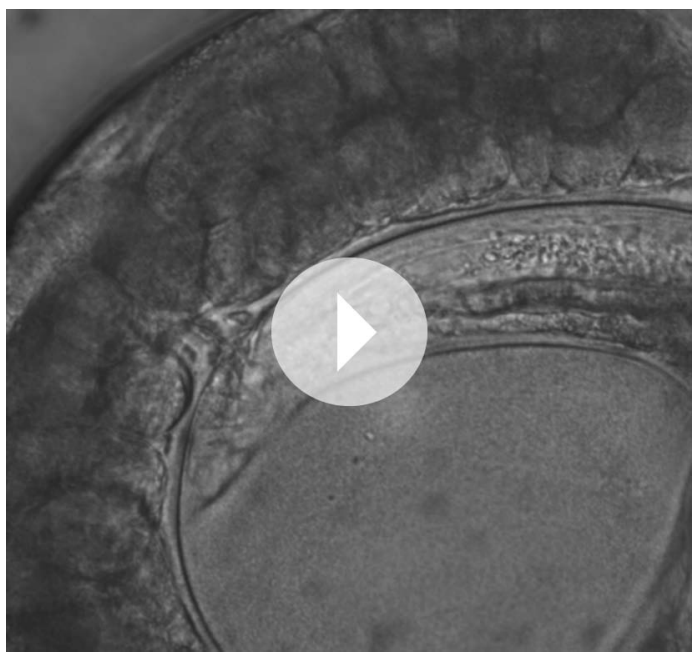

Video 3. 2-day-old sir-2.1(0) male's ejaculation. DOI: 10.7554/eLife.01730.009 genes, sir-2.1(0) males produced significantly more ATP at day 1. At day 2, wild-type ATP levels increased to match the level of sir-2.1(0) males. But at day 3, sir-2.1(0) males again accumulated more ATP (Figure 6B). These data suggest that sir-2.1(0) and older wild-type males might have an enhanced catabolism, consistent with the potential to generate more ROS.

Based on metabolic roles of PEPCK (Yang et al., 2009), up-regulation of pck genes could lead to excess glucose/glycogen in sir-2.1(0) males. Although similar amounts of glucose were detected in sir-2.1(0) and wild-type males (Figure 6-figure supplement 1), more glycogen was synthesized in the mutant (Figure $6 C$ ). In addition to gluconeogenesis, PEPCK catalysis of OAA to PEP is also a key step for the synthesis of glycerol-3-phosphate, which is used in triglyceride biosynthesis (Figure 6A) (Nye et al., 2008). Indeed, males lacking functional pck-2, but not pck-1 have less lipid content (Figure 6D).

Additionally, sir-2.1(0) males that lack pck-2, but not pck-1, showed reduced lipid staining (Figure 6D), indicating that pck-2 is necessary for the up-regulation of fat synthesis in sir-2.1(0). Taking together the real-time PCR results, accumulation of metabolic products and hypersensitivity to paraquat, we propose that in sir-2.1(0) males, glycolysis and fatty acid oxidation are up-regulated to provide excessive NADH to the electron transport chain. Since we also measured reduced expression of ETC components cytochrome c oxidase, more ROS might be generated via electron leak (Lee et al., 2010). However, we hypothesized that the enhanced expression of enzymes involved in anabolic processes might be a suboptimal self-compensatory mechanism to shunt excess pyruvate from being oxidized in the TCA cycle.

To test if the up-regulation of pck genes in sir-2.1(0) is a compensatory response, we assayed the mating potency of pck-1(0) and pck-2(0) single mutants and sir-2.1(0); pck-1(0) and pck-2(0); sir-2.1(0) double mutants. At day 1, sir-2.1(0) and pck-2(0) males mated comparable to wild type (Figure 6E). However at day 2, the potency of pck-2(0) males started to decline similarly to sir-2.1(0). In contrast, for males containing both sir-2.1(0) and pck-2(0), their mating potency dropped at day 1 (Figure 6E). This indicates that without pck-2, males that contain or lack sir-2.1 display accelerated behavioral decline. Similar to the requirement for functional pck-2, males that lack sir-2.1 also needed pck-1 to maintain their mating potency at day 1 ; however, pck-1 was not required for sir-2.1(+) males to mate efficiently at day 2 (Figure 6F).

Next, we reasoned that if excessive glycolysis contributes to the behavioral deterioration, artificially adding extra glucose to the males' media could accelerate their mating decline. To test this, we grew males on UV-killed-OP50 NGM plates supplemented with $2 \%$ glucose, from hatched larvae up to the adult age prior to behavioral decline, which is day 1 or day 2, for sir 2.1(0) and wild-type males, respectively. We found that the glucose reduced mating potency of 1-day-old sir-2.1(0), but not 2-day-old wild type (Figure 6G), indicating that wild type can cope with the extra glucose better than sir-2.1(0).

We hypothesized that unlike wild type, sir-2.1(0) males cannot efficiently respond to the oxidative stress generated by the enhanced catabolism. To test this, we used qRT-PCR to measure the mRNA levels of antioxidant genes: superoxide dismutase (sod-1, 2, 3, 4 and 5), catalase (ctl-1, 2), and glutathione transferase (gst-10 and gsto-1) relative to 1-day-old wild-type males (Figure 7). As expected, the expression of sod-1, sod-5, gst-10 and gsto-1 was reduced in 1-day-old sir-2.1(0) and 2-day-old wild type (Figure 7). For sod-2, day 1 expression was also reduced in sir-2.1(0), but this gene's expression increased in both wild type and sir-2.1(0) at day 2, possibly a stress response. For sod-3 and ctl-2, their day 1 expression was similar in both strains; however at day 2, sod-3 expression became higher and ctl-2 expression became lower in mutants. Finally, sir-2.1(0) males displayed an increased ctl-1 expression at day 1, which is also reported in antioxidant-compromised daf-16(0) mutant. The enhanced expression of $c t /-1$ is considered as an adaptive response (Yanase et al., 2002). These results indicate 
A
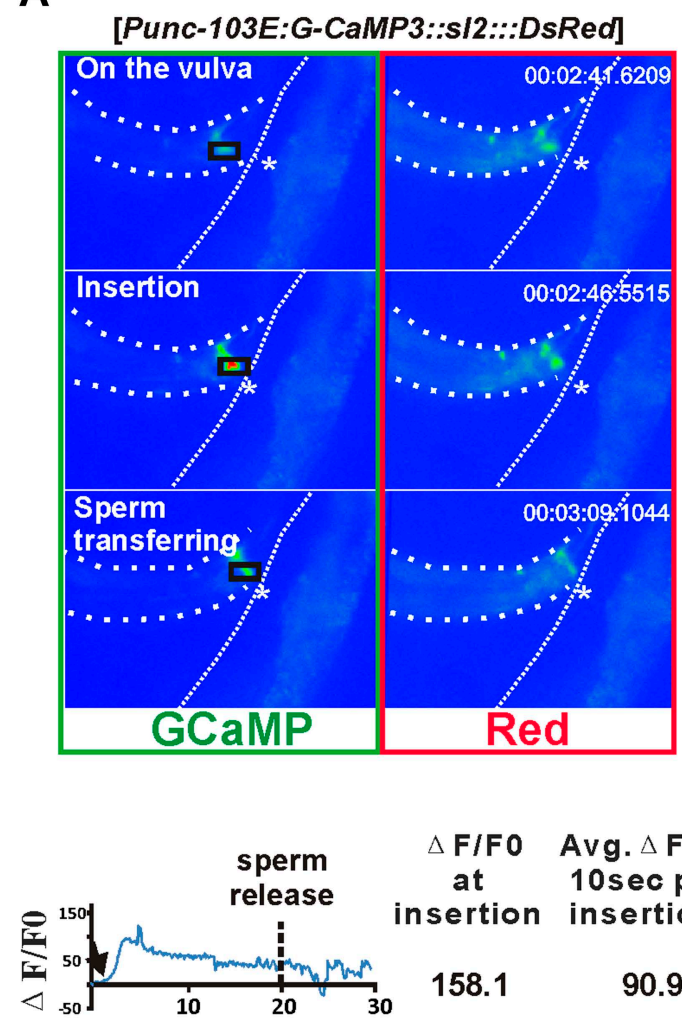

$\triangle F / F 0 \quad$ Avg. $\triangle F / F 0$

at 10 sec post insertion insertion

158.1
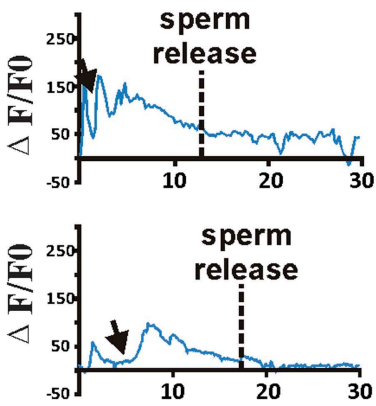

98.3

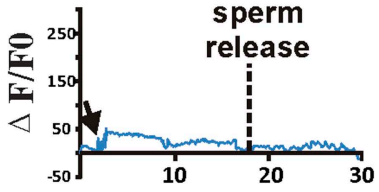

128.0

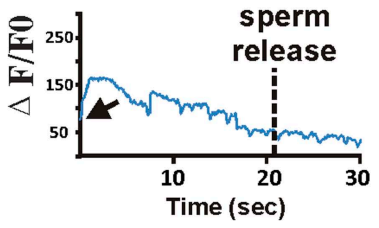

Average

$\triangle F / F 0$

$129+/-32.5 \quad 86.7+/-30.8$
B sir-2.1(0)

[Punc-103E:G-CaMP3::s/2:::DsRed]

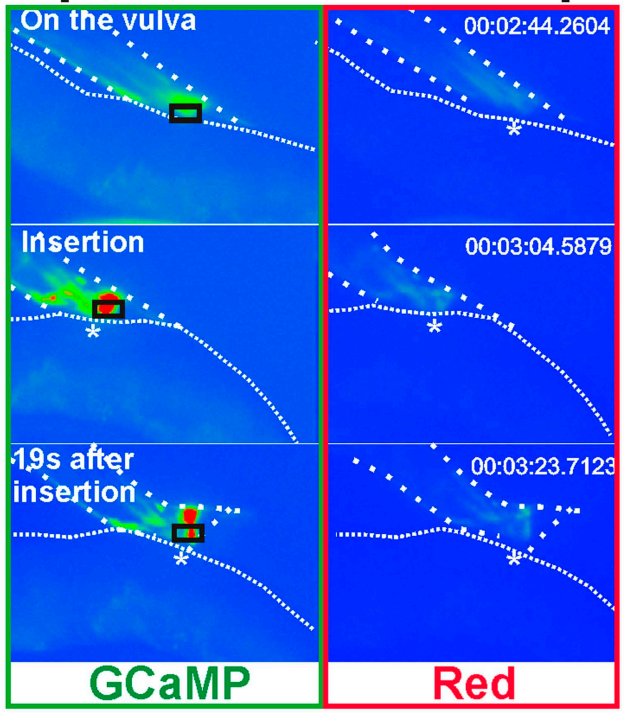

Dorsal protractor anal depressor
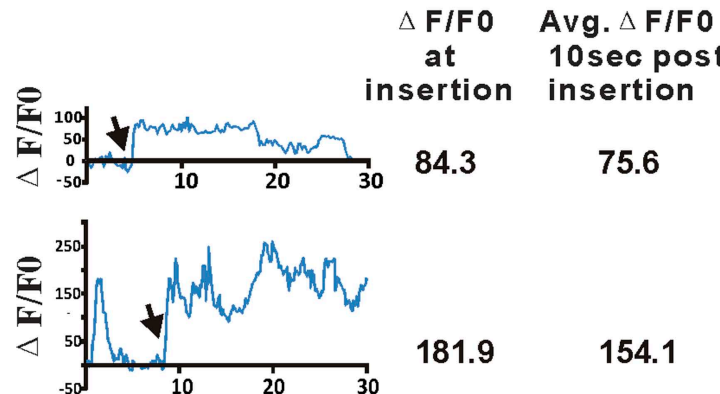

181.9

154.1

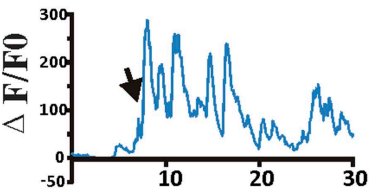

288.9

144.0

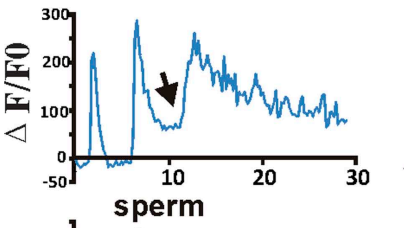

$261.0 \quad 153.6$

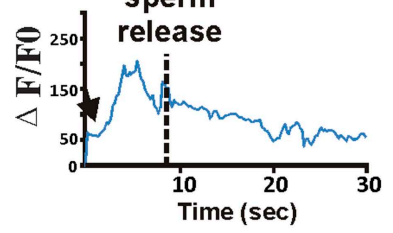

205.6

\section{Average}

$\triangle F / F 0$

Figure 3. $\mathrm{Ca}^{2+}$ imaging of spicule-associated muscles in mating males. Pseudo-colored images of $\mathrm{Ca}^{2+}$ in the spicule muscles of 2 -day-old wild-type and sir-2.1(0) males during mating $(\mathbf{A})$ and $(\mathbf{B})$ are representative frames to show the $\mathrm{Ca}^{2+}$ levels of the spicule-associated muscles during spicule insertion attempts, penetration and the start of sperm transfer ( $10 \mathrm{~s}$ after insertion for wild type) or $19 \mathrm{~s}$ after insertion (for sir-2.1(0) males). The asterisks indicate Figure 3. Continued on next page 
Figure 3. Continued

the hermaphrodite vulva. Below the images, the $\mathrm{Ca}^{2+}$ transients in the protractor and anal depressor muscles (indicated by the black rectangle in $\mathbf{A}$ and

B) are plotted for 5 individual wild-type (A) and sir-2.1(0) males (B), respectively.

DOI: 10.7554/eLife.01730.010

The following figure supplements are available for figure 3:

Figure supplement 1. A cartoon illustrating the contractile changes of the spicule-associated muscles during intromission and ejaculation behaviors of a 2-day-old wild-type and sir-2.1(0) male, respectively.

DOI: 10.7554/eLife.01730.011

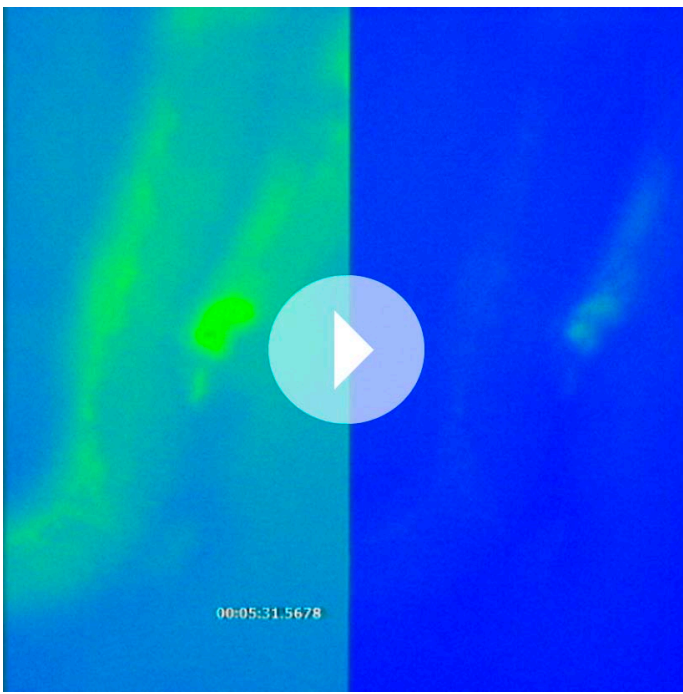

Video 4. $\mathrm{Ca}^{+}{ }^{+}$transient in a 2-day-old wild-type male. DOI: 10.7554/eLife.01730.012

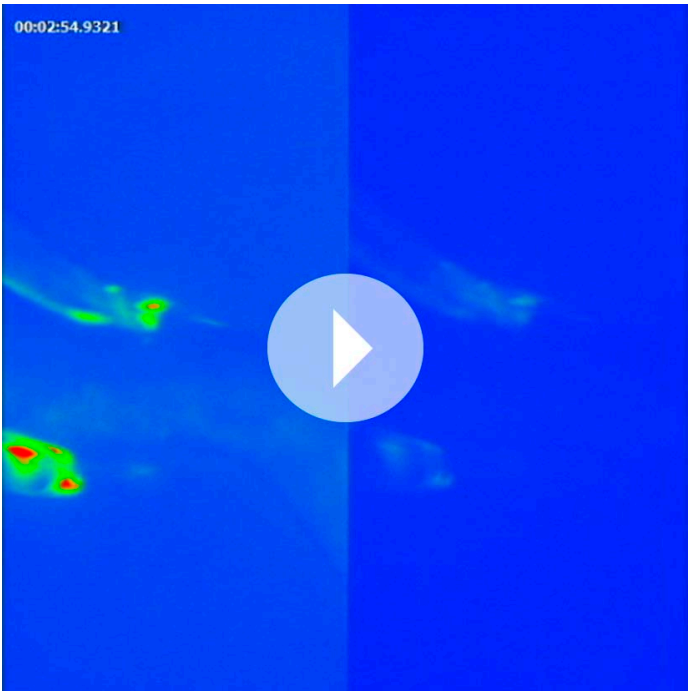

Video 5. Ca2+ transient in a 2-day-old sir-2.1(0) male. DOI: 10.7554/eLife.01730.013 SIR-2.1 does not amplify the positive effect of NAM (Figure 8D). Thus, we cannot exclude the possibility that NAM or possibly $\mathrm{NAD}^{+}$additionally promotes behavioral extension through mechanisms parallel to SIR-2.1 activity. that in addition to a potentially altered metabolism, which could generate excessive ROS, sir-2.1(0) males might also have a comprised antioxidant response, which is consistent with their hypersensitivity to excessive glucose intake (Figure 6G) and to the ROS generator (Figure 4A).

\section{Nicotinamide delays the deterioration of male mating behavior}

Based on the above results, one could hypothesize that increasing SIR-2.1 expression or activity might delay mating deterioration during aging. However, we found that transgenic overexpression of sir-2.1 does not improve the mating potency of 3-day-old wild type (Figure 8A). It is unlikely that the fusion with YFP disrupts SIR-2.1 function, because the same transgene can rescue the sir-2.1(0) phenotype. Thus, we speculate that up to a point, the expression level of sir-2.1 is not rate limiting for SIR-2.1 activity during early aging. However, one could also speculate that the normal endogenous levels of $\mathrm{NAD}^{+}$limit the function of SIR-2.1. To test this, we grew males in the presence of the $\mathrm{NAD}^{+}$precursor nicotinamide (NAM) at $200 \mu \mathrm{M}$ concentration (Houtkooper et al., 2010), and then conducted the mating potency. Indeed, NAM exposure significantly improves 3-day-old wild-type mating potency, but not 2-day-old sir-2.1(0) males (Figure 8B,C). This result is consistent with the idea that excess $\mathrm{NAD}^{+}$might stimulate SIR-2.1 activity. But additionally, excess $\mathrm{NAD}^{+}$might also reduce ROS production by relaxing the demand of oxidizing NADH back to NAD'; as a corollary to this possibility, the lack of excess NAM to positively affect the sir-2.1(0) male's behavior might be aggravated by the abnormally high expression of catabolic enzymes in the mutant males. To further test if overexpressing SIR-2.1 activity can promote mating behavior in older males, we exposed 3-day-old transgenic SIR-2.1 over-expressed males with exogenous NAM, but found that excess 


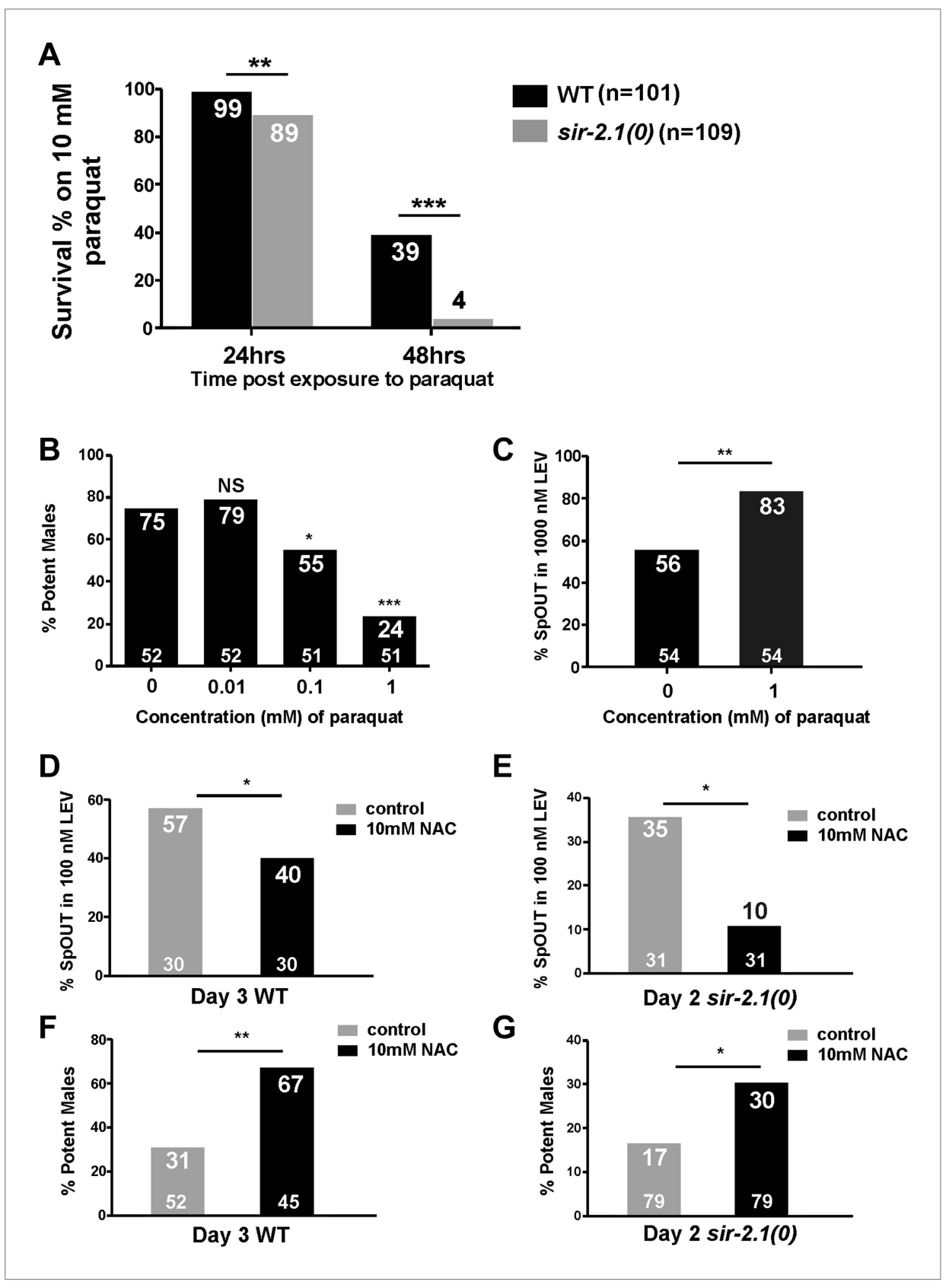

Figure 4. ROS contributes to the mating deterioration. (A) Survival rates of wild-type and sir-2.1(0) males on NGM containing $10 \mathrm{mM}$ paraquat at $24 \mathrm{hr}$ and $48 \mathrm{hr}$ post paraquat exposure. (B) Mating potency of 1-day-old wild-type males exposed to $0.01,0.1$, and $1 \mathrm{mM}$ paraquat. (C) The percentages of males with their spicules protruding out (SpOUT) in response to $1 \mu \mathrm{M}$ levamisole (LEV) after treatment with $1 \mathrm{mM}$ paraquat. (D-G) Exposing males to $\mathrm{N}$-acetyl-cystine (NAC) improves mating. The percentages of 3-day-old wild-type (D) and 2-day-old sir-2.1(0) (E) males that protrude their spicules out in response to $100 \mathrm{nM} L E V$ after NAC exposure. Mating potency of 3-day-old wild-type (F) and 2-day-old sir-2.1(0) (G) males after NAC exposure (Fisher's exact test). DOI: 10.7554/eLife.01730.014

\section{Discussion}

Behavioral decline at advanced age can be attributed to morphological degeneration, such as neuronal death and muscle sarcopenia (Herndon et al., 2002; Glenn et al., 2004). However, during early aging, prior to any significant cellular decay, physiological changes that affect neuronal and muscular functionality can contribute to the reduction of behavioral coordination (Salthouse, 2004). Male 


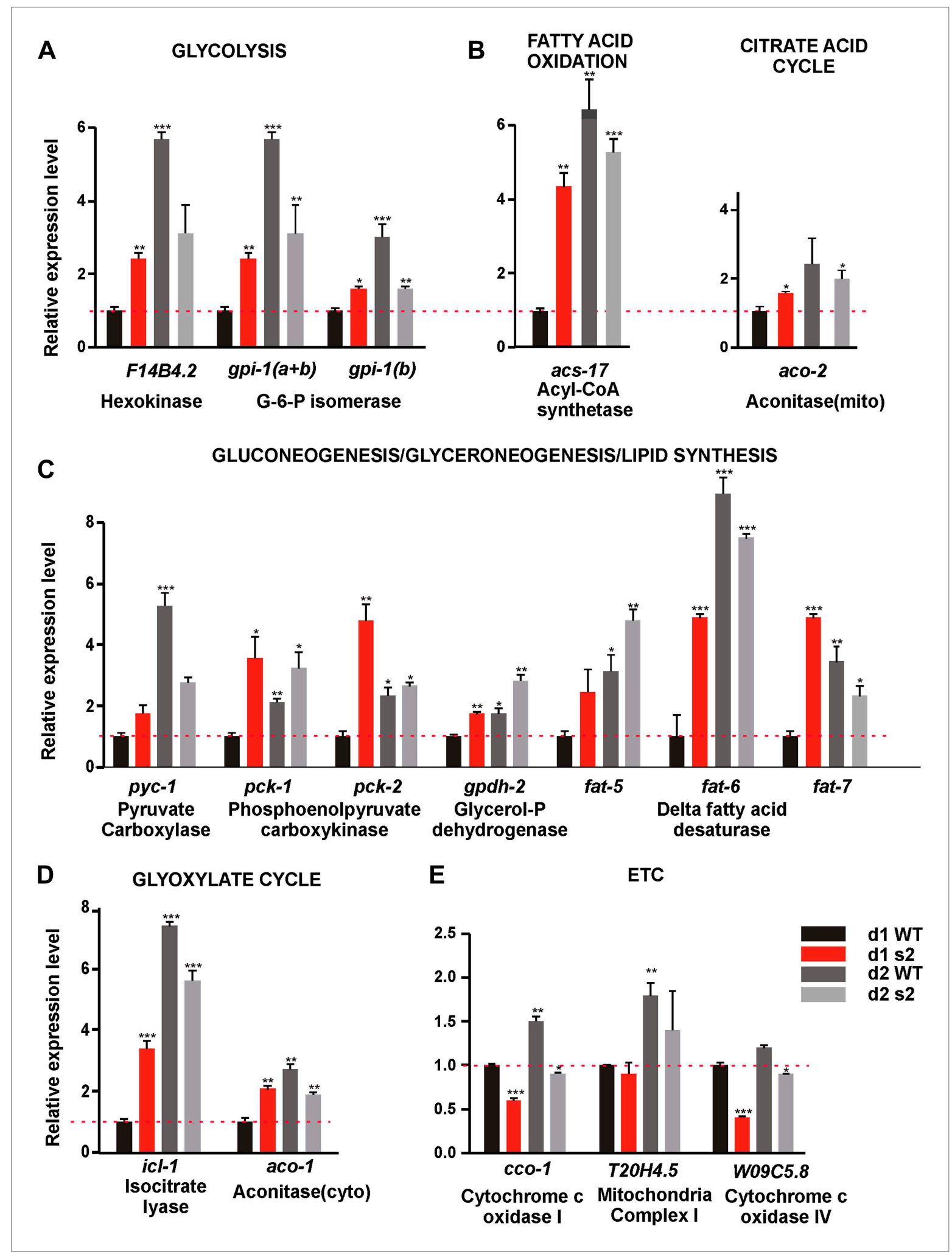

Figure 5. sir-2.1(0) males have altered expression of metabolic genes. Relative mRNA expression level of genes involved in metabolic processes such as glycolysis (A), TCA cycle (B), fatty acid oxidation(C), Gluconeogenesis/glyceroneogenesis/lipid synthesis (D), Glyoxylate cycle (E), and ETC (F) in 2-day-old wild type, 1-day-old, and 2-day-old sir-2.1(0) males relative to 1-day-old wild type. d1 WT refers to day1 wild type; d2 WT refers to day 2 wild type; d1 s2 refers to day1 sir-2.1(0); d2 s2 refers to day 2 sir-2.1(0) (unpaired t-test compared to 1-day-old wild type). 
A

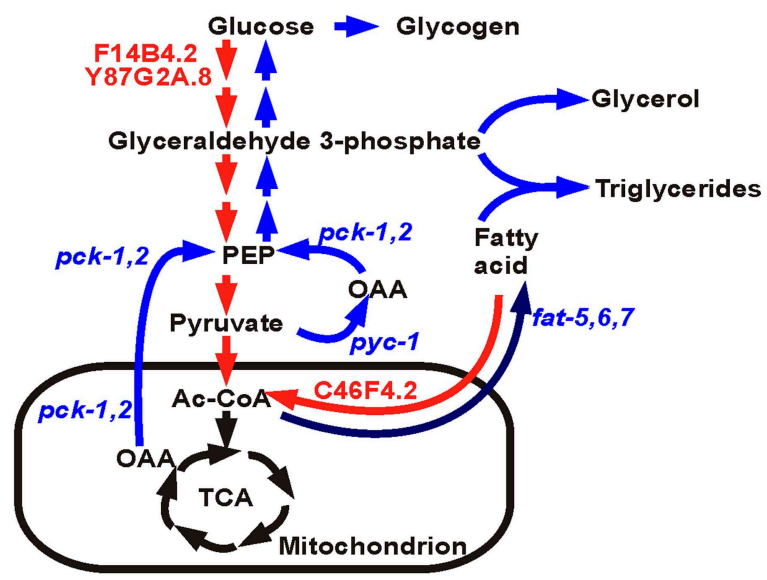

D

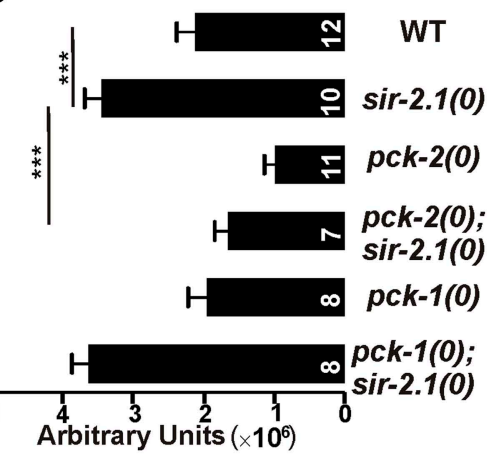

Day 1

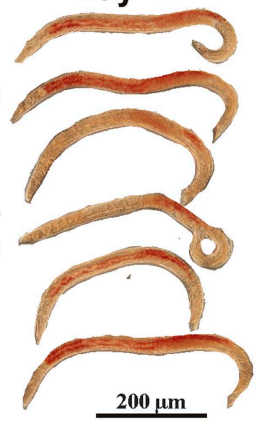

$\mathbf{F}$

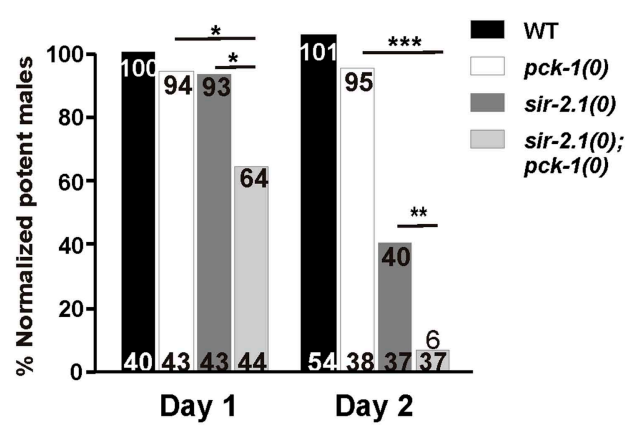

B

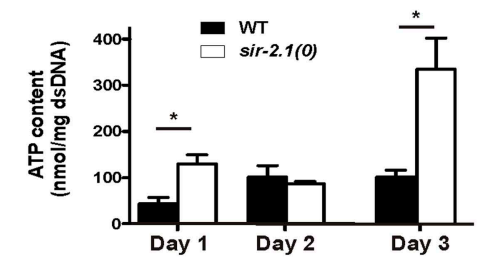

C

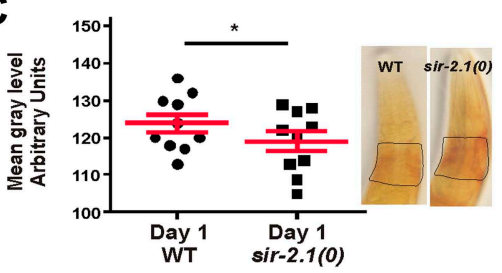

E

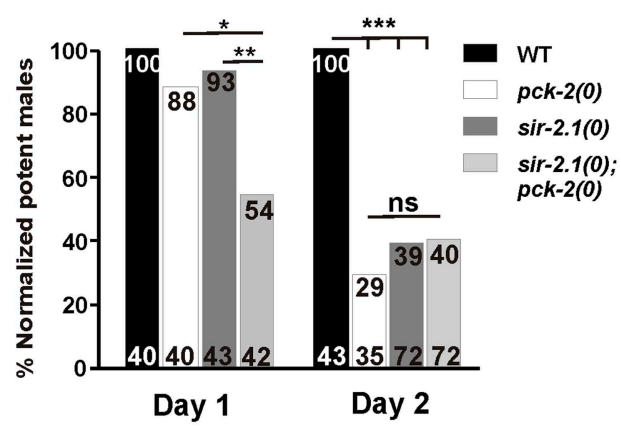

G

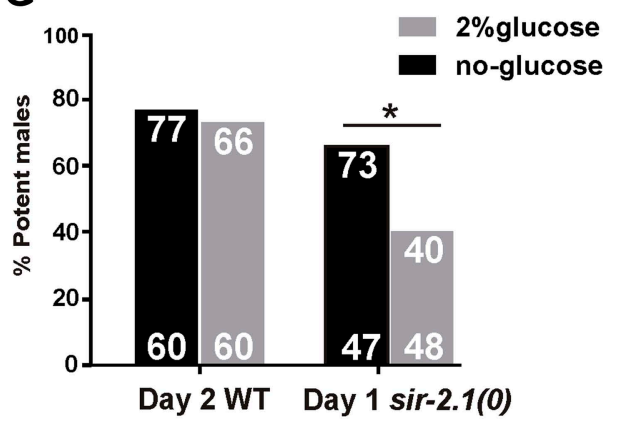

Figure 6. sir-2.1(0) males might have enhanced metabolism. (A) Schematic illustration of main metabolic enzymes which have altered expression in sir-2.1(0) males. Red arrows indicate catabolic pathways. Blue arrows indicate anabolic pathways. (B) ATP content measured in 1, 2 and 3-day-old wild-type and sir-2.1(0) males. (C) Glycogen staining in 1-day-old wild type and sir-2.1(0). The glycogen staining level is quantified by measuring the mean gray level of the ROI indicated on the top right corner. The mean gray level is inversely correlated with the iodine stain. (D) Oil Red $O$ staining of wild type and mutant C. elegans males. (E) sir-2.1(+) and sir-2.1(0) need pck-2 to maintain their mating at day 2 and day 1 respectively. All percentages of mating potency are normalized to that of 1-day-old wild-type male. (F) sir-2.1(0) requires pck-1 to maintain their mating at day 1 and day 2, while sir-2.1(+) males do not need pck-2 to maintain their mating at either day 1 or day 2. (G) $2 \%$ glucose reduces 1-day-old sir-2.1(0) mating potency, but not 2-day-old wild-type males (Fisher's exact test).

DOI: 10.7554/eLife.01730.016

The following figure supplements are available for figure 6:

Figure supplement 1. The level of glucose content is similar between 1-day-old sir-2.1(0) and wild-type males.

DOI: 10.7554/eLife.01730.017

mating in C. elegans is a complex behavior that requires coordination of multiple motor systems to impregnate the hermaphrodite (Liu et al., 2011; Correa et al., 2012). Previously, we showed that male mating behavior declines significantly during early aging. Wild type cannot mate well at 


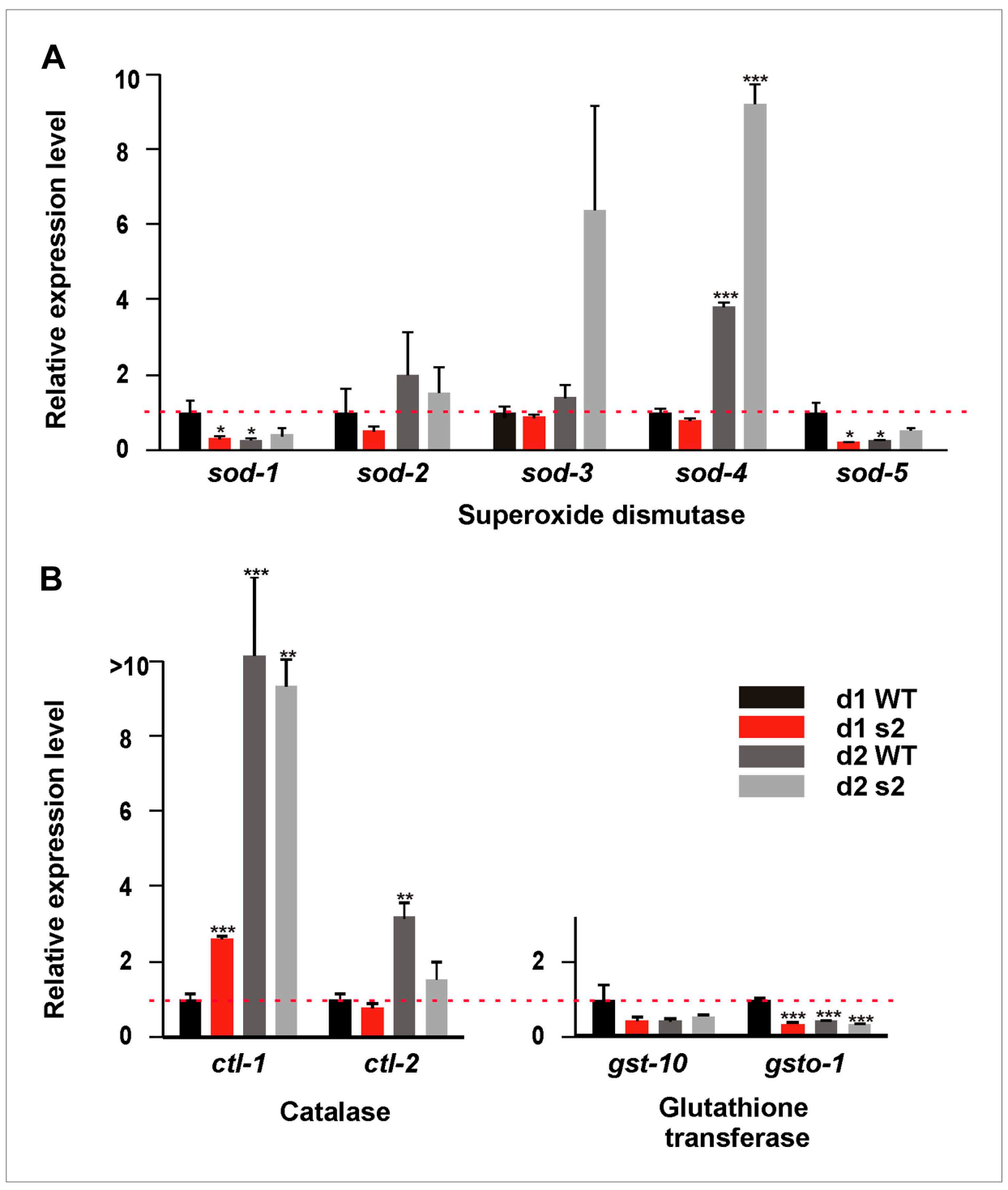

Figure 7. sir-2.1(0) males have compromised expression of anti-oxidant genes. Relative mRNA expression level of anti-oxidant genes superoxide dismutase (A), catalase (B), and glutathione transferase (C) in 1, 2-day-old wild type and sir-2.1(0) males (unpaired t-test).

DOI: 10.7554/eLife.01730.018

adulthood day 3, and this decline is correlated with an increased excitability in the sex circuitry (Guo et al., 2012). Similar to aging males, a recent study using hermaphrodites showed that synaptic transmission in the locomotion circuit is enhanced after 5 days of adulthood (Mulcahy et al., 2013). Thus, it is possible that during early aging, the neuromuscular systems of both sexes become hyperexcitable. However, the faster decline in mating suggests that the male reproductive circuitry is more sensitive to age-related physiological changes.

We found that SIR-2.1 is a modulator of behavior and is required to maintain mating in aging males. 1-day-old sir-2.1(0) males can mate similarly to wild type, suggesting that sir-2.1 is not essential for mating. However, unlike wild-type males, the mating ability of sir-2.1(0) prematurely drops at day 2. This is due to hyperexcitability of the reproductive circuitry that coordinates spicule intromission and ejaculation. The hyperexcitability of the spicule muscles causes the male proctodeum to block the connection between the vas deferens and the cloacal opening, which indirectly obstructs the transfer of sperm. The mutant phenotype resembles the behavioral, physiological, and pharmacological changes that occur in older wild-type males. This indicates that in wild type, SIR-2.1 maintains the functional excitability of the intromission and ejaculation circuit, possibly by slowing down the 
A

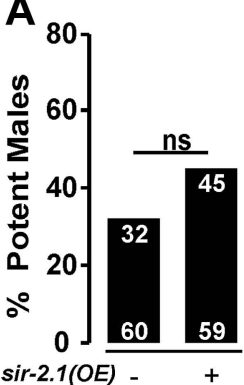

3-day-old WT

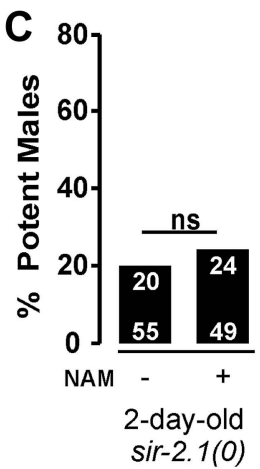

B
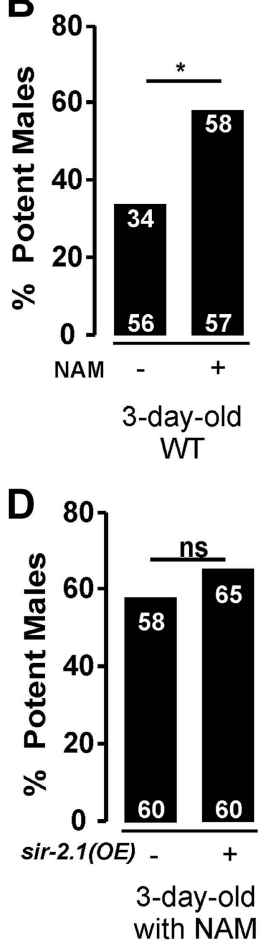

Figure 8. Exogenous nicotinamide improves mating during aging. sir-2.1 overexpression cannot increase mating potency of 3-day-old wild type (A). However, feeding with a $N A D^{+}$precursor nicotinamide (NAM) significantly improve the mating potency of 3-day-old wild type (B) but not 2-day-old sir-2.1(0) males (C). Overexpression of sir-2.1 cannot further promote the effect of exogenous NAM (D).

DOI: 10.7554/eLife.01730.019

ratio of $\mathrm{NAD}^{+}$to $\mathrm{NADH}$ in wild type The phenomenon of altering $\mathrm{NAD}^{+}$to $\mathrm{NADH}$ levels in vertebrate cells is shown to reduce the activity of SIRT1 (Braidy et al., 2011). Decreased activity of SIR-2.1 will not only aggravate a bias towards catabolism, but will also decrease the levels of ROS scavengers. This is consistent with a hermaphrodite study, which showed that sir-2.1 overexpression can protect the organism from ROS, possibly via HCF-1 and FOXO/DAF-16, to regulate the expression of stress response genes (Rizki et al., 2011).

Although our qPCR analyses suggest that enhanced catabolism might be occurring in 1-day-old of sir-2.1(0) and 2-day-old wild-type males, their mating ability could be facilitated via anabolic compensatory mechanisms. In addition to enhanced expression of catabolic genes, mRNAs encoding enzymes such as pyruvate carboxylase and phosphoenolpyruvate carboxykinase (PEPCK) appeared to be also up-regulated. This could be a likely reason for why the males contain more measurable lipids and glycogen. We propose that the up-regulation of anabolic process is a self-compensatory mechanism to divert carbon from the TCA cycle (Figure 9). sir-2.1(0) males that are mutant for PEPCK genes, lose their ability to generate fat and fail to mate efficiently on day 1. Likewise sir-2.1 (+) males with a mutation in PEPCK genes also display premature mating decline. We hypothesize that anabolic pathways could act as a homeostatic mechanism to reduce ROS production. This idea raises the possibility that obesity as a phenotype might be a compensatory mechanism to alleviate the effects of other underlying metabolic dysfunctions. Indeed, by switching of glycolysis to gluconeogenesis has been shown to be a potential strategy to cure hepatocarcinoma (Ma et al., 2013). A recent study showed that diabetic patients, who are not obese, have higher mortality than overweight ones (Carnethon et al., 2012). 


\section{1-day-old WT}

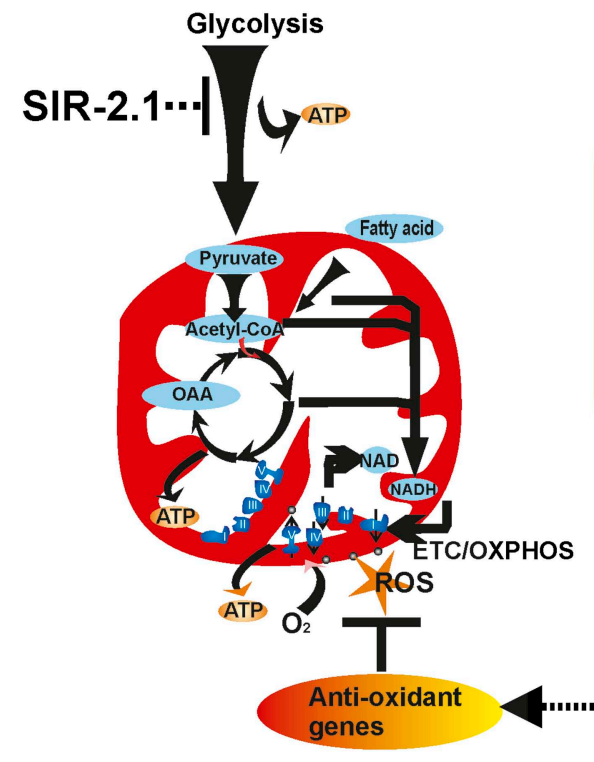

SIR-2.1

\section{2-day-old WT}

1-day-old sir-2.1(0)

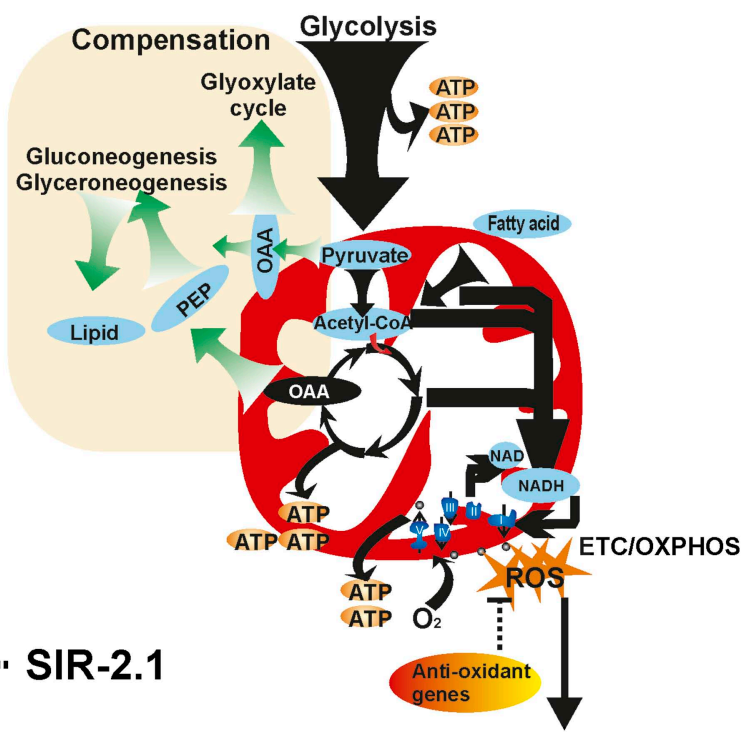

Hyper-excitability of genital circuits on day 2 for sir-2.1(0) and day 3-4 for WT
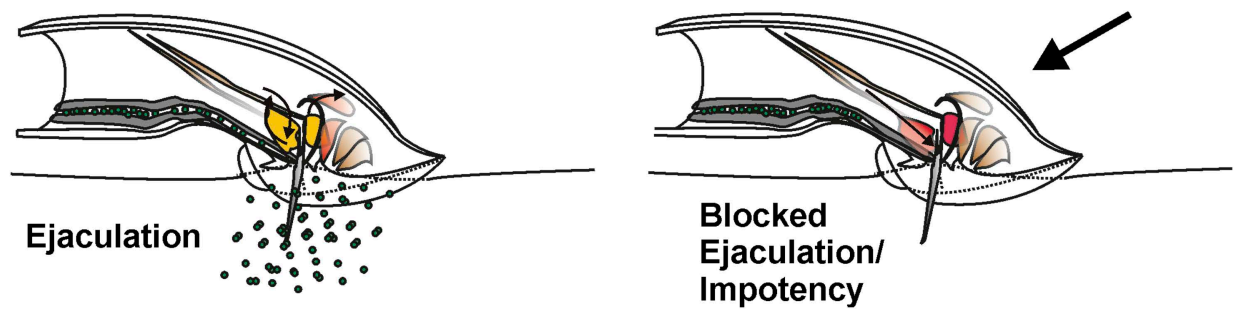

Figure 9. A cartoon of the metabolism and behavior that occurs in wild-type and sir-2.1(0) males during early aging. For successful reproductive behavior, SIR-2.1 is required to maintain proper carbon flow to meet the male's energy demands and balance the generation of ROS. In 1-day-old old sir-2.1(0) males, catabolism such as glycolysis and fatty acid oxidation is enhanced, and consequently, oxidative phosphorylation and generation of ROS are also increased. Without SIR-2.1, ROS accumulation by day 2 of adulthood can lead to hyperexcitability of the male's genital neuromuscular circuitry. This results in blocked ejaculation and impotency. It is possible that in 2- to 3-day-old wild-type males, the NAD+-dependent SIR-2.1 activity declines due to a lower ratio of NAD to NADH; thus older wild-type males might have a similar physiology as 1-day-old sir-2.1(0) males.

DOI: 10.7554/eLife.01730.020

Another study showed that a lifestyle intervention focusing on weight loss did not reduce the rate of cardiovascular events in obese adults with type II diabetes (Look AHEAD Research Group, 2013), challenging the traditional viewpoint that obesity is a major contributor to metabolic disorders.

The free radical aging theory states that aging is the result of free radical-induced molecular damages (Harman, 1956). Although this notion is challenged by the hormesis theory, which posits that moderate amounts of physiological or environmental insults can reinforce cellular processes that reduce stress-induced damage (Afanas'ev, 2010; Schulz et al., 2007), artificially applied ROS is reported to change the excitability of cultured neurons and muscles through chemically damaging ion channels (Danson and Paterson, 2006). Therefore, aspects of the free radical theory of aging might still apply to physiological changes in neural muscular systems and behavioral decay. Consistent with this, we showed here that genetic and environmental conditions, which can lead to 
oxidative stress, caused increased excitability of the male mating circuitry and behavioral decay during early aging.

There is not a strict correlation between oxidative stress and changes in the electrical properties of neurons and muscles. Oxidation of different types of ion channels changes their conductance and alters the cell's excitability (Annunziato et al., 2002). One C. elegans study indicates that oxidative stress reduces cell excitability by increasing the conductance of $\mathrm{K}^{+}$channels (Sesti et al., 2010). Another mammalian study showed that oxidative stress hyperpolarizes the resting potential, but extends the duration of the action potential in cardiac ganglion (Whyte et al., 2009). Mitochondria ROS has been shown to trigger $\mathrm{Ca}^{2+}$ increases in the pulmonary arterial myocytes (Waypa et al., 2002, 2006). In a study using glia, L-type voltage-gated $\mathrm{Ca}^{2+}$ channels (L-VGCC) were found to be a target of ROS. After modification by ROS, their conductance of $\mathrm{Ca}^{2+}$ was increased (Bond and Greenfield, 2007). Different from vertebrate skeletal muscles, L-VGCC in C. elegans propagates action potentials, and the entry of external $\mathrm{Ca}^{2+}$ directly promotes excitation-contraction coupling (Lee et al., 1997; Maryon et al., 1998). Previous work showed that L-VGCC EGL-19 in C. elegans is required for sustained tonic contraction of the copulatory spicule muscles (Garcia et al., 2001). Therefore, oxidation of L-VGCC in C. elegans might contribute to the increased excitability of mating circuits. Other major targets of ROS are voltage-gated $\mathrm{K}^{+}$channels. In C. elegans, oxidation of KVS-1 slows down its inactivation, leading to hyperpolarization and sensory function loss (Cai and Sesti, 2009). The ERG-like $\mathrm{K}^{+}$channel UNC-103 is a major excitability regulator of the sex circuit (Reiner et al., 2006). Although there is no report of oxidative modification on UNC-103, human encoded H-ERG channels can be activated by oxidative stress, so that cells become hyperpolarized and less excitable (Cui and Zhang, 2013). Considering that ROS increases the excitability of the mating circuit, it is possible that L-VGCC is more prone to be oxidized than $\mathrm{K}^{+}$channels in male reproductive cells.

\section{Materials and methods}

\section{Strains and culture conditions}

Worms were grown at $20^{\circ} \mathrm{C}$ on nematode growth media (NGM) plates seeded with E. coli strain OP50, except for strains containing the pha-1(e2123), which were maintained at $15^{\circ} \mathrm{C}$. The alleles used in this work included: lite-1(ce314) on LGX; pck-2(ok2586) on LGl; pck-1(ok2098), pha-1(e2123) (Schnabel and Schnabel, 1990), unc-64(e240) (Brenner, 1974) on LGIII; sir-2.1(ok434) on LGIV; and him-5(e1490) (Hodgkin et al., 1979) on LGV. The males were generated by the him-5(e1490) mutation. Males containing only this mutation are referred to as wild-type; him-5(e1490) males have been shown to mate efficiently as wild type (Hodgkin, 1983). pck-2(ok2586), pck-1(ok2098), and sir-2.1(ok434) were generated by the C. elegans Gene Knockout Consortium. sir-2.1(ok434) animals were out-crossed 4 times with the him-5(e1490) strain. The deletion in sir-2.1(0) was detected through PCR using primers listed in the Supplementary file 2.

Altered mediums used here included NGM medium containing glucose (2\%), paraquat, $\mathrm{N}$-acetylcystine (NAC) (Sigma, St. Louis, MO), and nicotinamide (NAM) (Sigma) respectively. The latter three were added at the indicated concentration just before pouring the plates. OP50 used for the special medium containing glucose and NAC was UV-killed and concentrated to make sure the worms were not food deprived. We assume that the animals ingest these compounds as they feed on $E$. coli or absorb them through their cuticle.

\section{Transgenic constructs}

DNA primers are listed in the Supplementary file 2. The sir-2.1 genomic sequence, plus 2 kb upstream of its ATG, was PCR-amplified from N2 DNA. The PCR product was digested and ligated between the Sphl and Sall sites of pSX322YFP to obtain the plasmid pXG5. To obtain promoters for driving sir-2.1 expression, the sir-2.1 endogenous promoter was removed via PCR-mutagenesis from pXG5 to construct pXG6. The Gateway ATTR cassette frame A was inserted in front of the sir- 2.1 genomic sequence to make the destination clone pXG7. Plasmids ( $X$ XG8, pXG9, and pXG11) that promote neuronal, muscular, and intestinal expression of sir-2.1 were obtained through Gateway LR reactions between pXG7 and pLR35 (Paex-3) (LeBoeuf et al., 2007), pLR22 (Plev-11) (Gruninger et al., 2008)

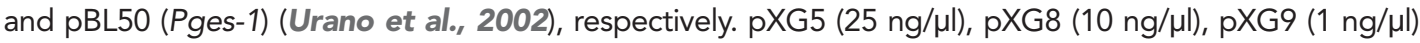
or pXG11 (50 ng/ $\mu \mathrm{l})$ were injected to sir-2.1(0) hermaphrodites and transgenic animals were selected 
via YFP fluorescence. pXG5 (50 ng/ $\mu \mathrm{l})$ was injected into wild-type hermaphrodites to obtain strains with overexpression of sir-2.1 (referred as sir-2.1(OE)).

\section{Behavioral assays}

The mating potency assay was performed as described in Guo et al. (2012). Briefly, L4 males were isolated from hermaphrodites. One male and one pha-1(e2123) hermaphrodite were co-transferred to a 5 -mm-diameter OP50 mating lawn at $20^{\circ} \mathrm{C}$, which is a restrictive temperature for pha-1(e2123) to sire viable self-progeny. This allowed us to score the mating potency of males, as only cross-progenies would develop to adulthood.

To quantify different parameters of mating, we observed, up to $5 \mathrm{~min}$, copulations between males and paralyzed unc-64(e240) hermaphrodites. The ability of males to sense the vulva was calculated by counting the number of times he stopped at the vulva divided by the total number of times he stopped and/or passed by the vulva. The turning quality was calculated as: the number of smooth turns (defined as the male tail keeping contact with hermaphrodite and turning without hesitation) divided by the total number of turns. Ejaculation assays were conducted in two ways. We directly observed sperm transfer after spicule insertion, and determined if sperm drained into the unc-64(e240) hermaphrodite's uterus. Additionally, cross-progeny were counted 1-2 days after spicules insertion.

\section{Lifespan and stress resistance assays}

Lifespan assay was conducted as described in Guo et al. (2012). L4 males were isolated and raised 20 per plate. The males that can respond to gentle touch with a platinum wire were counted and transferred to new plates every day. Males that dried on the wall of the Petri plate were censored from the assay on the day they died.

To assess the sensitivity to paraquat, L4 males were transferred to plates containing $10 \mathrm{mM}$ paraquat and scored at 24 and $48 \mathrm{hr}$.

\section{In vitro sperm activation assay}

Sperm activation assays were done according to L'Hernault and Roberts (1995); Smith and Stanfield (2011). Briefly, three 2-day-old males, isolated at L4 stage, were cut at the posterior portion with a needle in $20 \mu \mathrm{l}$ sperm media $(50 \mathrm{mM}$ Hepes, $\mathrm{pH} 7.0,45 \mathrm{mM} \mathrm{NaCl}, 25 \mathrm{mM} \mathrm{KCl}, 1 \mathrm{mM} \mathrm{MgSO}$, and $5 \mathrm{mM} \mathrm{CaCl}_{2}$ ) freshly supplemented with polyvinylprolidone (PVP) 40,000 molecular weight (Sigma) and the activator pronase (Roche, Indianapolis, IN) at the final concentration of $10 \mathrm{mg} / \mathrm{ml}$ and $500 \mu \mathrm{g} / \mathrm{ml}$ on a slide. A coverslip with a thin layer of Vaseline applied around the edge was put on the top of the sperm media to form a chamber over the sperm. After 5-min incubation, activated sperm with a pseudopod and inactive ones were counted using a compound scope fitted with a 100X objective. Approximately, 50-60 sperm cells were counted in each sample section.

\section{Drug tests}

Arecoline (ARE) and levamisole (LEV) were dissolved in water at the concentration of $100 \mathrm{mM}$, and then diluted accordingly, as described in Liu et al. (2007). Briefly, $50 \mu \mathrm{M}$ of ARE, $1 \mu \mathrm{M}$ and $500 \mathrm{nM}$ of LEV for 1, 2-day-old males, and 100 nM LEV for 3-day-old males were used. Males were introduced to $1 \mathrm{ml}$ drug baths and scored if they protruded their spicules for more than $5 \mathrm{~s}$ within 5 min of drug exposure.

\section{$\mathrm{Ca}^{2+}$ imaging}

$\mathrm{Ca}^{2+}$ imaging was conducted and analyzed as described in Guo et al. (2012). $50 \mathrm{ng} / \mu \mathrm{l}$ pLR289[Punc103E:G-CaMP3::s/2:::DsRed] (Correa et al., 2012) and $50 \mathrm{ng} / \mu \mathrm{lpBX1(Granato} \mathrm{et} \mathrm{al.,} \mathrm{1994)} \mathrm{were}$ co-injected into pha-1(e2123); him-5(e1490); lite-1(ce314) hermaphrodites to generate the extrachromosomal array rgEx566[Punc-103E:G-CaMP3::s/2:::DsRed]. rgEx566 was crossed into pha-1(e2123); him-5(e1490); sir-2.1(0); lite-1(ce314) hermaphrodites. Fluorescence signals from G-CaMP3 and DsRed were recorded simultaneously during the copulations of 2-day-old wild-type and sir-2.1(0) males with paralyzed hermaphrodites containing the transgene rgEx431[Phsp-16:egl-2(gf); Punc-103E:DsRed].

\section{Real-time PCR}

300 day 1 and day 2 adult males were accumulated over a period of time. RNA was extracted by Trizol, and cDNA was synthesized by SuperScript II (Life technology, Grand Island, NY) using around $2 \mu \mathrm{g}$ total RNA, as described in LeBoeuf and Garcia (2012). The RT-qPCR reactions were performed using 
BIO-RAD CFX96 real-time system and SsoFast EvaGreen supermix. 11 candidate reference genes were tested to see whether their expression changed from day 1 and day 2 in both wild-type and sir-2.1 males (Hoogewijs et al., 2008); from our analyses, act-1 and gpd-2 were selected as the reference to normalize the expression of the metabolic genes. Many of the primers used to detect the expression of metabolic enzymes are described in Castelein et al. (2008). Other primers for additional metabolic and antioxidant stress genes are listed in the Supplementary file 2. Three replicates were conducted on the same RNA samples. We used the t-test to determine which mRNA transcripts in sir-2.1(0) males were significantly different from their cognate wild-type transcripts.

\section{ATP, glucose, glycogen, and lipid measurements}

To measure ATP and glucose, 100 males were collected at different ages, frozen and thawed three times. The worms were homogenized, and the supernatant was collected and measured using an ATP determination Kit (Life technology) and the Glucose Oxidase Assay Kit (Life technology). The ATP and glucose were normalized to the amount of dsDNA quantified by picoGreen (Life technology).

To stain glycogen, 1-day-old sir-2.1(0) and wild-type virgin males were transferred to $2 \%$ agar pads. The pads containing both genotypes were then placed over a bottle of iodine crystals for $30 \mathrm{~s}$ (Frazier and Roth, 2009). The pictures were taken by a Leica compound scope mounted with OLYMPUS DP70 camera. The RGB images were then converted to 16-bit gray scale, and the mean gray levels of the isthmus regions were measured using the SimplePCl image quantification software (Hamamatsu, Janpan). The mean gray level was reversely correlated with the red signal.

Oil Red O staining was done according to O'Rourke et al. (2009). Briefly, males were collected and washed with PBS, and then fixed with Modified Ruvkuns witches brew (MRWB) buffer containing 1\% paraformaldehyde (PFA) for $1 \mathrm{hr}$. Worms were then washed with PBS and suspended in 60\% isopropanol for $15 \mathrm{~min}$ at room temperature. The $60 \%$ isopropanol was removed and worms were bathed in $60 \%$ Red Oil O staining solution. The RGB images were taken by a Leica compound scope mounted with OLYMPUS DP70 camera. The images were quantified by ImageJ according to Mehlem et al. (2013).

\section{Acknowledgements}

We thank D Gualberto for technical assistance, and B LeBoeuf, C Jee, P Correa, L Zhang, Y Liu, and X Chen for discussion of the project and critical reading of the manuscript. C. elegans strains were provided by the Caenorhabditis Genetic Center. This work is supported by the Howard Hughes Medical Institute.

\section{Additional information}

Funding

\begin{tabular}{ll} 
Funder & Author \\
\hline Howard Hughes Medical Institute & L René García
\end{tabular}

The funder had no role in study design, data collection

and interpretation, or the decision to submit the work for publication.

Author contributions

XG, Conception and design, Acquisition of data, Analysis and interpretation of data, Drafting or revising the article; LRG, Conception and design, Analysis and interpretation of data, Drafting or revising the article

\section{Additional files}

Supplementary files

- Supplementary file 1. Expression levels of metabolic enzymes though real-time PCR. d1 WT refers to day1 wild type; d2 WT refers to day 2 wild type; d1 s2 refers to day1 sir-2.1(0); d2 s2 refers to day 2 sir-2.1(0).

DOI: 10.7554/eLife.01730.021

- Supplementary file 2. Primers used in this study.

DOI: 10.7554/eLife.01730.022 


\section{References}

Afanas'ev I. 2010. Signaling and damaging functions of free radicals in aging-free radical theory, hormesis, and TOR. Aging Disease 1:75-88.

Aggarwal NT, Makielski JC. 2013. Redox control of cardiac excitability. Antioxidants \& Redox Signaling 18:432-468. doi: 10.1089/ars.2011.4234.

Annunziato L, Parmaccione A, Cataldi M, Secondo A, Castaldo P, Di Renzo G, Taglialatela M. 2002. Modulation of ion channels by reactive oxygen and nitrogen species: a pathophysiological role in brain aging? Neurobiology of Aging 23:819-834. doi: 10.1016/S0197-4580(02)00069-6.

Bamps S, Wirtz J, Savory FR, Lake D, Hope IA. 2009. The Caenorhabditis elegans sirtuin gene, sir-2.1, is widely expressed and induced upon caloric restriction. Mechanisms of Ageing and Development 130:762-770. doi: 10.1016/j.mad.2009.10.001.

Berdichevsky A, Viswanathan M, Horvitz HR, Guarente L. 2006. C. elegans SIR-2.1 interacts with 14-3-3 proteins to activate DAF-16 and extend life span. Cell 125:1165-1177. doi: 10.1016/j.cell.2006.04.036.

Bond CE, Greenfield SA. 2007. Multiple cascade effects of oxidative stress on astroglia. Glia 55:1348-1361. doi: 10.1002/glia.20547.

Braidy N, Guillemin GJ, Mansour H, Chan-Ling T, Poljak A, Grant R. 2011. Age related changes in NAD plus metabolism oxidative stress and Sirt1 activity in wistar rats. PLOS ONE 6:e19194. doi: 10.1371/journal.pone.0019194.

Brenner S. 1974. The genetics of Caenorhabditis elegans. Genetics 77:71-94.

Burnett C, Valentini S, Cabreiro F, Goss M, Somogyvari M, Piper MD, Hoddinott M, Sutphin GL, Leko V, McElwee JJ, Vazquez-Manrique RP, Orfila AM, Ackerman D, Au C, Vinti G, Riesen M, Howard K, Neri C, Bedalov A, Kaeberlein M, Soti C, Partridge L, Gems D. 2011. Absence of effects of Sir2 overexpression on lifespan in C. elegans and Drosophila. Nature 477:482-485. doi: 10.1038/nature10296.

Cai SQ, Sesti F. 2009. Oxidation of a potassium channel causes progressive sensory function loss during aging. Nature Neuroscience 12:611-617. doi: 10.1038/nn.2291.

Carnethon MR, De Chavez PJD, Biggs ML, Lewis CE, Pankow JS, Bertoni AG, Golden SH, Liu K, Mukamal KJ, Campbell-Jenkins B, Dyer AR. 2012. Association of weight status with mortality in adults with incident diabetes. JAMA: The Journal of the American Medical Association 308:581-590. doi: 10.1001/jama.2012.9282.

Castelein N, Hoogewijs D, De Vreese A, Braeckman BP, Vanfleteren JR. 2008. Dietary restriction by growth in axenic medium induces discrete changes in the transcriptional output of genes involved in energy metabolism in Caenorhabditis elegans. Biotechnol Journal 3:803-812. doi: 10.1002/biot.200800003.

Correa P, LeBoeuf B, Garcia LR. 2012. C. elegans dopaminergic D2-like receptors delimit recurrent cholinergicmediated motor programs during a goal-oriented behavior. PLOS Genetics 8:e1003015. doi: 10.1371/journal. pgen.1003015.

Cui Z, Zhang S. 2013. Regulation of the human ether-a-go-go-related gene (hERG) channel by Rab4 protein through neural precursor cell-expressed developmentally down-regulated protein 4-2 (Nedd4-2). The Journal of Biological Chemistry 288:21876-21886. doi: 10.1074/jbc.M113.461715.

Danson EJ, Paterson DJ. 2006. Reactive oxygen species and autonomic regulation of cardiac excitability. Journal of Cardiovascular Electrophysiology 17(suppl 1):S104-S112. doi: 10.1111/j.1540-8167.2006.00391.x.

Federico A, Cardaioli E, Da Pozzo P, Formichi P, Gallus GN, Radi E. 2012. Mitochondria, oxidative stress and neurodegeneration. Journal of the Neurological Sciences 322:254-262. doi: 10.1016/j.jns.2012.05.030.

Flowers MT, Ntambi JM. 2008. Role of stearoyl-coenzyme A desaturase in regulating lipid metabolism. Current Opinion in Lipidology 19:248-256. doi: 10.1097/MOL.0b013e3282f9b54d.

Frazier HN, Roth MB. 2009. Adaptive sugar provisioning controls survival of $C$. elegans embryos in adverse environments. Current Biology 19:859-863. doi: 10.1016/j.cub.2009.03.066.

Garcia LR, Mehta P, Sternberg PW. 2001. Regulation of distinct muscle behaviors controls the C. elegans male's copulatory spicules during mating. Cell 107:777-788. doi: 10.1016/S0092-8674(01)00600-6.

Garcia LR, Sternberg PW. 2003. Caenorhabditis elegans UNC-103 ERG-like potassium channel regulates contractile behaviors of sex muscles in males before and during mating. The Journal of Neuroscience 23:2696-2705.

Glenn CF, Chow DK, David L, Cooke CA, Gami MS, Iser WB, Hanselman KB, Goldberg IG, Wolkow CA. 2004. Behavioral deficits during early stages of aging in Caenorhabditis elegans result from locomotory deficits possibly linked to muscle frailty. The Journals of Gerontology. Series A, Biological Sciences and Medical Sciences 59:1251-1260. doi: 10.1093/gerona/59.12.1251.

Gomes AP, Price NL, Ling AJ, Moslehi JJ, Montgomery MK, Rajman L, White JP, Teodoro JS, Wrann CD, Hubbard BP, Mercken EM, Palmeira CM, de Cabo R, Rolo AP, Turner N, Bell EL, Sinclair DA. 2013. Declining $\mathrm{NAD}(+)$ induces a pseudohypoxic state disrupting nuclear-mitochondrial communication during aging. Cell 155:1624-1638. doi: 10.1016/j.cell.2013.11.037.

Gottlieb S, Esposito RE. 1989. A new role for a yeast transcriptional silencer gene, Sir2, in regulation of recombination in ribosomal DNA. Cell 56:771-776. doi: 10.1016/0092-8674(89)90681-8.

Granato M, Schnabel H, Schnabel R. 1994. Pha-1, a selectable marker for gene-transfer in C. elegans. Nucleic Acids Research 22:1762-1763. doi: 10.1093/nar/22.9.1762.

Gruninger TR, Gualberto DG, Garcia LR. 2008. Sensory perception of food and insulin-like signals influence seizure susceptibility. PLOS Genetics 4:e1000117. doi: 10.1371/journal.pgen.1000117.

Guo X, Navetta A, Gualberto DG, Garcia LR. 2012. Behavioral decay in aging male C. elegans correlates with increased cell excitability. Neurobiology of Aging 33:e1485-e1423. doi: 10.1016/j.neurobiolaging.2011.12.016. 
Harman D. 1956. Aging: a theory based on free radical and radiation chemistry. Journal of Gerontology 11:298-300. doi: 10.1093/geronj/11.3.298.

Herndon LA, Schmeissner PJ, Dudaronek JM, Brown PA, Listner KM, Sakano Y, Paupard MC, Hall DH, Driscoll M. 2002. Stochastic and genetic factors influence tissue-specific decline in ageing C. elegans. Nature 419:808-814. doi: 10.1038/nature01135.

Hodgkin J. 1983. Male phenotypes and mating efficiency in CAENORHABDITIS ELEGANS. Genetics 103:43-64.

Hodgkin J, Horvitz HR, Brenner S. 1979. Nondisjunction mutants of the nematode CAENORHABDITIS ELEGANS. Genetics 91:67-94.

Hoogewijs D, Houthoofd K, Matthijssens F, Vandesompele J, Vanfleteren JR. 2008. Selection and validation of a set of reliable reference genes for quantitative sod gene expression analysis in $C$. elegans. BMC Molecular Biology 9:9. doi: 10.1186/1471-2199-9-9.

Houtkooper RH, Canto C, Wanders RJ, Auwerx J. 2010. The secret life of NAD+: an old metabolite controlling new metabolic signaling pathways. Endocrine Reviews 31:194-223. doi: 10.1210/er.2009-0026.

Houtkooper RH, Pirinen E, Auwerx J. 2012. Sirtuins as regulators of metabolism and healthspan. Nature Reviews. Molecular Cell Biology 13:225-238. doi: 10.1038/nrm3293.

Hursting SD, Lavigne JA, Berrigan D, Perkins SN, Barrett JC. 2003. Calorie restriction, aging, and cancer prevention: mechanisms of action and applicability to humans. Annual Review of Medicine 54:131-152. doi: 10.1146/annurev.med.54.101601.152156.

Jin K. 2010. Modern biological theories of aging. Aging Disease 1:72-74.

Kaeberlein M, McVey M, Guarente L. 1999. The SIR2/3/4 complex and SIR2 alone promote longevity in Saccharomyces cerevisiae by two different mechanisms. Genes \& Development 13:2570-2580. doi: 10.1101/ gad.13.19.2570.

Kenyon CJ. 2010. The genetics of ageing. Nature 464:504-512. doi: 10.1038/nature08980.

L'Hernault SW, Roberts TM. 1995. Cell biology of nematode sperm. Methods in Cell Biology 48:273-301.

LeBoeuf B, Garcia LR. 2012. Cell excitability necessary for male mating behavior in Caenorhabditis elegans is coordinated by interactions between big current and ether-a-go-go family $\mathrm{K}(+)$ channels. Genetics 190:1025-1041. doi: 10.1534/genetics.111.137455.

LeBoeuf B, Gruninger TR, Garcia LR. 2007. Food deprivation attenuates seizures through CaMKII and EAG K+ channels. PLOS Genetics 3:1622-1632. doi: 10.1371/journal.pgen.0030156.

LeBoeuf B, Guo X, Garcia LR. 2011. The effects of transient starvation persist through direct interactions between CaMKII and ether-a-go-go K+ channels in C. elegans males. Neuroscience 175:1-17. doi: 10.1016/j. neuroscience.2010.12.002.

Lee RY, Lobel L, Hengartner M, Horvitz HR, Avery L. 1997. Mutations in the alpha1 subunit of an L-type voltage-activated $\mathrm{Ca}^{2+}$ channel cause myotonia in Caenorhabditis elegans. The EMBO Journal 16:6066-6076. doi: 10.1093/emboj/16.20.6066.

Lee SJ, Hwang AB, Kenyon C. 2010. Inhibition of respiration extends C. elegans life span via reactive oxygen species that increase HIF-1 activity. Current Biology: CB 20:2131-2136. doi: 10.1016/j.cub.2010.10.057.

Liu Y, LeBeouf B, Guo X, Correa PA, Gualberto DG, Lints R, Garcia LR. 2011. A cholinergic-regulated circuit coordinates the maintenance and bi-stable states of a sensory-motor behavior during Caenorhabditis elegans male copulation. PLOS Genetics 7:e1001326.

Liu Y, LeBoeuf B, Garcia LR. 2007. G alpha(q)-coupled muscarinic acetylcholine receptors enhance nicotinic acetylcholine receptor signaling in Caenorhabditis elegans mating behavior. The Journal of Neuroscience 27:1411-1421. doi: 10.1523/JNEUROSCI.4320-06.2007.

Look AHEAD Research Group. 2013. Cardiovascular effects of intensive lifestyle intervention in type 2 diabetes. The New England Journal of Medicine 369:145-154. doi: 10.1056/NEJMoa1212914.

Ma R, Zhang W, Tang K, Zhang H, Zhang Y, Li D, Li Y, Xu P, Luo S, Cai W, Ji T, Katirai F, Ye D, Huang B. 2013. Switch of glycolysis to gluconeogenesis by dexamethasone for treatment of hepatocarcinoma. Nature Communications 4:2508. doi: 10.1038/ncomms3508.

Maryon EB, Saari B, Anderson P. 1998. Muscle-specific functions of ryanodine receptor channels in Caenorhabditis elegans. Journal of Cell Science 111:2885-2895.

Mehlem A, Hagberg CE, Muhl L, Eriksson U, Falkevall A. 2013. Imaging of neutral lipids by oil red O for analyzing the metabolic status in health and disease. Nature Protocols 8:1149-1154. doi: 10.1038/nprot.2013.055.

Merksamer PI, Liu YF, He WJ, Hirschey MD, Chen D, Verdin E. 2013. The sirtuins, oxidative stress and aging: an emerging link. Aging-Us 5:144-150.

Mulcahy B, Holden-Dye L, O'Connor V. 2013. Pharmacological assays reveal age-related changes in synaptic transmission at the Caenorhabditis elegans neuromuscular junction that are modified by reduced insulin signalling. The Journal of Experimental Biology 216:492-501. doi: 10.1242/jeb.068734.

Murphy MP. 2009. How mitochondria produce reactive oxygen species. Biochemical Journal 417:1-13. doi: 10.1042/BJ20081386.

Nye CK, Hanson RW, Kalhan SC. 2008. Glyceroneogenesis is the dominant pathway for triglyceride glycerol synthesis in vivo in the rat. Journal of Biological Chemistry 283:27565-27574. doi: 10.1074/jbc. M804393200.

O'Rourke EJ, Soukas AA, Carr CE, Ruvkun G. 2009. C. elegans major fats are stored in vesicles distinct from lysosome-related organelles. Cell Metabolism 10:430-435. doi: 10.1016/j.cmet.2009.10.002.

Reiner DJ, Weinshenker D, Tian H, Thomas JH, Nishiwaki K, Miwa J, Gruninger T, Leboeuf B, Garcia LR. 2006. Behavioral genetics of Caenorhabditis elegans unc-103-encoded erg-like $\mathrm{K}(+)$ channel. Journal of Neurogenetics 20:41-66. doi: 10.1080/01677060600788826. 
Rizki G, Iwata TN, Li J, Riedel CG, Picard CL, Jan M, Murphy CT, Lee SS. 2011. The evolutionarily conserved longevity determinants HCF-1 and SIR-2.1/SIRT1 collaborate to regulate DAF-16/FOXO. PLoS Genetics 7:e1002235. doi: 10.1371/journal.pgen.1002235.

Salthouse TA. 2004. What and when of cognitive aging. Current Directions in Psychological Science 13:140-144. doi: 10.1111/j.0963-7214.2004.00293.x.

Satoh A, Stein L, Imai S. 2011. The role of mammalian sirtuins in the regulation of metabolism, aging, and longevity. Handbook of Experimental Pharmacology 206:125-162. doi: 10.1007/978-3-642-21631-2_7.

Schnabel R, Schnabel H. 1990. Early determination in the C. elegans embryo: a gene, cib-1, required to specify a set of stem-cell-like blastomeres. Development 108:107-119.

Schulz TJ, Zarse K, Voigt A, Urban N, Birringer M, Ristow M. 2007. Glucose restriction extends Caenorhabditis elegans life span by inducing mitochondrial respiration and increasing oxidative stress. Cell Metabolism 6:280-293. doi: 10.1016/j.cmet.2007.08.011.

Sesti F, Liu S, Cai SQ. 2010. Oxidation of potassium channels by ROS: a general mechanism of aging and neurodegeneration? Trends in Cell Biology 20:45-51. doi: 10.1016/j.tcb.2009.09.008.

Smith JR, Stanfield GM. 2011. TRY-5 is a sperm-activating protease in Caenorhabditis elegans seminal fluid. PLOS Genetics 7:e1002375. doi: 10.1371/journal.pgen.1002375.

Taglialatela M, Castaldo P, lossa S, Pannaccione A, Fresi A, Ficker E, Annunziato L. 1997. Regulation of the human ether-a-gogo related gene (HERG) $\mathrm{K}^{+}$channels by reactive oxygen species. Proceedings of the National Academy of Sciences of the United States of America 94:11698-11703. doi: 10.1073/pnas.94.21.11698.

Tan KT, Luo SC, Ho WZ, Lee YH. 2011. Insulin/IGF-1 receptor signaling enhances biosynthetic activity and fat mobilization in the initial phase of starvation in adult male C. elegans. Cell Metabolism 14:390-402. doi: 10.1016/j.cmet.2011.06.019.

Tian L, Hires SA, Mao T, Huber D, Chiappe ME, Chalasani SH, Petreanu L, Akerboom J, McKinney SA, Schreiter ER, Bargmann Cl, Jayaraman V, Svoboda K, Looger LL. 2009. Imaging neural activity in worms, flies and mice with improved GCaMP calcium indicators. Nature Methods 6:875-881. doi: 10.1038/nmeth.1398.

Tissenbaum HA, Guarente L. 2001. Increased dosage of a sir-2 gene extends lifespan in Caenorhabditis elegans. Nature 410:227-230. doi: 10.1038/35065638.

Urano F, Calfon M, Yoneda T, Yun C, Kiraly M, Clark SG, Ron D. 2002. A survival pathway for C. elegans with a blocked unfolded protein response. The Journal of Cell Biology 158:639-646. doi: 10.1083/jcb.200203086.

Van Gilst MR, Hadjivassiliou H, Yamamoto KR. 2005. A Caenorhabditis elegans nutrient response system partially dependent on nuclear receptor NHR-49. Proceedings of the National Academy of Sciences of the United States of America 102:13496-13501. doi: 10.1073/pnas.0506234102.

Viswanathan M, Guarente L. 2011. Regulation of Caenorhabditis elegans lifespan by sir-2.1 transgenes. Nature 477:E1-E2. doi: 10.1038/nature10440.

Walker AK, Yang FJ, Jiang KR, Ji JY, Watts JL, Purushotham A, Boss O, Hirsch ML, Ribich S, Smith JJ, Israelian K, Westphal CH, Rodgers JT, Shioda T, Elson SL, Mulligan P, Najafi-Shoushtari H, Black JC, Thakur JK, Kadyk LC, Whetstine JR, Mostoslavsky R, Puigserver P, Li X, Dyson NJ, Hart AC, Näär AM. 2010. Conserved role of SIRT1 orthologs in fasting-dependent inhibition of the lipid/cholesterol regulator SREBP. Genes \& Development 24:1403-1417. doi: 10.1101/gad.1901210.

Wang RH, Kim HS, Xiao C, Xu X, Gavrilova O, Deng CX. 2011. Hepatic Sirt1 deficiency in mice impairs mTorc2/ Akt signaling and results in hyperglycemia, oxidative damage, and insulin resistance. The Journal of Clinical Investigation 121:4477-4490. doi: 10.1172/JCl46243.

Waypa GB, Guzy R, Mungai PT, Mack MM, Marks JD, Roe MW, Schumacker PT. 2006. Increases in mitochondrial reactive oxygen species trigger hypoxia-induced calcium responses in pulmonary artery smooth muscle cells. Circulation Research 99:970-978. doi: 10.1161/01.RES.0000247068.75808.3f.

Waypa GB, Marks JD, Mack MM, Boriboun C, Mungai PT, Schumacker PT. 2002. Mitochondrial reactive oxygen species trigger calcium increases during hypoxia in pulmonary arterial myocytes. Circulation Research 91:719-726. doi: 10.1161/01.RES.0000036751.04896.F1.

Webster BR, Lu Z, Sack MN, Scott I. 2012. The role of sirtuins in modulating redox stressors. Free Radical Biology \& Medicine 52:281-290. doi: 10.1016/j.freeradbiomed.2011.10.484.

Whyte KA, Hogg RC, Dyavanapalli J, Harper AA, Adams DJ. 2009. Reactive oxygen species modulate neuronal excitability in rat intrinsic cardiac ganglia. Autonomic Neuroscience: Basic \& Clinical 150:45-52. doi: 10.1016/j. autneu.2009.04.005.

Yanase S, Yasuda K, Ishii N. 2002. Adaptive responses to oxidative damage in three mutants of Caenorhabditis elegans (age-1, mev-1 and daf-16) that affect life span. Mechanisms of Ageing and Development 123: 1579-1587. doi: 10.1016/S0047-6374(02)00093-3.

Yang JQ, Kalhan SC, Hanson RW. 2009. What is the metabolic role of phosphoenolpyruvate carboxykinase? Journal of Biological Chemistry 284:27025-27029. doi: 10.1074/jbc.R109.040543. 